\title{
Probing the scalar potential via double Higgs boson production at hadron colliders
}

\author{
Sophia Borowka, ${ }^{a}$ Claude Duhr, ${ }^{a, b}$ Fabio Maltoni, ${ }^{b, c}$ Davide Pagani, ${ }^{d}$ \\ Ambresh Shivaji ${ }^{b, e}$ and Xiaoran Zhao $^{b}$ \\ a Theoretical Physics Department, CERN, \\ CH-1211 Geneva 23, Switzerland \\ ${ }^{b}$ Centre for Cosmology, Particle Physics and Phenomenology (CP3), \\ Université Catholique de Louvain, \\ B-1348 Louvain-la-Neuve, Belgium \\ ${ }^{c}$ Dipartimento di Fisica e Astronomia, Università di Bologna and INFN, Sezione di Bologna, \\ via Irnerio 46, 40126 Bologna, Italy \\ ${ }^{d}$ Technische Universität München, \\ James-Franck-Str. 1, D-85748 Garching, Germany \\ ${ }^{e}$ Department of Physical Sciences, Indian Institute of Science Education and Research (IISER), \\ Mohali Knowledge City, Sector 81 S.A.S. Nagar, PO-Manauli PIN-140306, Punjab, India \\ E-mail: sophia.borowka@cern.ch, claude.duhr@cern.ch, \\ fabio.maltoni@uclouvain.be, davide.pagani@tum.de, \\ ambresh.shivaji@uclouvain.be, xiaoran.zhao@uclouvain.be
}

ABSTRACT: We present a sensitivity study on the cubic and quartic self couplings in double Higgs production via gluon fusion at hadron colliders. Considering the relevant operators in the Standard Model Effective Field Theory up to dimension eight, we calculate the dominant contributions up to two-loop level, where the first dependence on the quartic interaction appears. Our approach allows to study the independent variations of the two self couplings and to clearly identify the terms necessary to satisfy gauge invariance and to obtain UV-finite results order by order in perturbation theory. We focus on the $b \bar{b} \gamma \gamma$ signature for simplicity and provide the expected bounds for the cubic and quartic self couplings at the $14 \mathrm{TeV}$ LHC with $3000 \mathrm{fb}^{-1}$ (HL-LHC) and for a future $100 \mathrm{TeV}$ collider (FCC-100) with $30 \mathrm{ab}^{-1}$. We find that while the HL-LHC will provide very limited sensitivity on the quartic self coupling, precision measurements of double Higgs production at a FCC-100 will offer the opportunity to set competitive bounds. We show that combining information from double and triple Higgs production leads to significantly improved prospects for the determination of the quartic self coupling.

Keywords: NLO Computations, Phenomenological Models

ARXIV EPRINT: 1811.12366 


\section{Contents}

1 Introduction 1

2 Calculation $\quad 4$

2.1 Parametrisation of $\lambda_{3}$ and $\lambda_{4}$ effects 4

2.2 Organisation of the calculation 8

$\begin{array}{lll}2.3 & \text { Two-loop non-factorisable terms } & 10\end{array}$

$\begin{array}{lll}2.3 .1 & \text { Reduction to form factors } & 10\end{array}$

$\begin{array}{ll}\text { 2.3.2 Numerical evaluation of the form factors } & 12\end{array}$

$\begin{array}{lll}2.3 .3 & \text { Grids for phase-space integration } & 14\end{array}$

3 Numerical results $\quad 15$

4 Constraints on the Higgs self couplings $\quad 20$

$\begin{array}{ll}4.1 \text { General set up } & 20\end{array}$

4.2 Scenario $1 \quad 22$

4.3 Scenario $2 \quad 24$

$\begin{array}{llr}5 & \text { Conclusion } & 27\end{array}$

$\begin{array}{lr}\text { A Cut efficiency } & 28\end{array}$

B Fit details $\quad 29$

C Topologies of the integral expressions from non-factorisable two-loop contributions

\section{Introduction}

Since the discovery in 2012 by the ATLAS and CMS collaboration [1, 2], the Large Hadron Collider (LHC) has already disclosed an impressive amount of information on the properties of the resonance at $125 \mathrm{GeV}$, confirming so far the expectations of the Standard Model (SM). The new particle is a narrow scalar [3, 4], interacting with (third generation) fermions and vector bosons with a strength proportional to the mass of the particle [5,6]. All the expected main production and decay modes have been observed [1, 2, 7-12]. Future runs at the LHC and future colliders will provide new information (such as the coupling to second generation fermions) and higher accuracy on the known couplings. The current measurements already indicate that New Physics (NP) effects cannot substantially affect the couplings of the Higgs boson with vector bosons and third generation fermions, placing the scale of NP well above the electroweak symmetry breaking scale. 
The situation, however, is very different for the scalar potential on which we have not gained any relevant information so far and which is therefore largely unexplored. The reason is simply that the scalar potential, whose shape is ultimately responsible for Electroweak Symmetry Breaking (EWSB), can be probed only by measuring the Higgs self couplings. At hadron and lepton colliders, a direct sensitivity on the cubic or quartic Higgs self couplings can be achieved only via the simultaneous production of two or three Higgs bosons, respectively. Due to the smallness of the corresponding SM cross sections (in the gluon fusion channel, which is the dominant one, at $13 \mathrm{TeV}$ they are around $30 \mathrm{fb}$ for $p p \rightarrow H H$ and $0.05 \mathrm{fb}$ for $p p \rightarrow H H H$ ) these processes have not yet been observed at the LHC; an observation would have been a clear sign of beyond-the-SM (BSM) physics. Therefore, the study of the Higgs self couplings is currently not only far from the precision level but also very challenging for the future.

In the case of double Higgs production, only exclusion limits are currently available and the most stringent result has been obtained by the ATLAS collaboration. Combining three different analyses $\left(4 b, b \bar{b} \tau \tau\right.$, and $b \bar{b} \gamma \gamma$ signatures) based on $27.5-36.1 \mathrm{fb}^{-1}$ of data accumulated at $13 \mathrm{TeV}$ [13-16], cross sections larger than 6.7 times the $\mathrm{SM}$ one can be excluded. This limit translates into the bound $-5.0 \lambda_{3}^{\mathrm{SM}}<\lambda_{3}<12.1 \lambda_{3}^{\mathrm{SM}}$, where $\lambda_{3}$ is the cubic coupling and $\lambda_{3}^{\mathrm{SM}}$ is its SM prediction. With a collected luminosity of $300 \mathrm{fb}^{-1}$, or even with $3000 \mathrm{fb}^{-1}$ in the case of the High-Luminosity (HL) option, it is not still clear if the observation of SM production can be achieved. Although many phenomenological studies have been performed [17-39], the best experimental predictions for HL-LHC only provide upper limits on the SM cross sections. On the other hand, precise predictions are already available for this process; concerning QCD corrections, the full NLO [40-42], the NLO matched with shower effects [43-45], the NNLO in the large top-mass limit [46-49] and even improved with the full NLO [50] have been calculated. Besides reducing the scale dependence, QCD higher-order corrections increase the LO cross section by roughly a factor of 2 .

In the case of $\lambda_{4}$, the prospects are very uncertain. At the LHC, inferring information from triple Higgs production will be extremely challenging [51, 52]. Its cross section is very small and depends on the quartic interaction very weakly. Even a future $100 \mathrm{TeV}$ proton-proton collider will need a considerable amount of integrated luminosity in order to obtain rather loose bounds [53-56]. Also for this process precise predictions are already available [57-59].

Given the current and expected future results, new complementary strategies for the determination of the Higgs self couplings would be desirable. Recently, the possibility of probing the cubic Higgs self coupling $\lambda_{3}$ via precision measurements of single Higgs production channels at future lepton colliders [60] and at the LHC and future hadron colliders $[61,62]$ has been suggested, exploiting the fact that next-to-leading order (NLO) EW corrections to the single Higgs production and decay modes involve $\lambda_{3}$. The turning point for the possibility of determining the cubic interaction from single Higgs production measurements at the LHC has been the understanding that the different production channels depend on $\lambda_{3}$ in a very different way and that the effects are differential, the sensitivity being enhanced at threshold [62]. Even though the expected effects are small, a competitive 
sensitivity can be obtained by combining globally information from single Higgs measurements, total cross sections as well as distributions [62]. Since then, considerable effort has been invested in studying the feasibility of this strategy: predictions for the differential distributions for all the Higgs production channels have become available [63, 64], and studies with more general (and realistic) scenarios for the existence of anomalous Higgs interactions [64-67] have appeared, also in combination with the direct double Higgs information [65-68]. Following the same logic, the $\lambda_{3}$ bounds have been extracted also from EW precision observables $[69,70]$. It is now clear that the indirect determination of $\lambda_{3}$ via precision measurements is expected to provide comparable bounds to those that are currently obtained via the direct searches for double Higgs production. Very recently it has been proposed that double Higgs production could be exploited for probing the quartic Higgs self coupling $\lambda_{4}$ via precise measurements [68,71]. The first studies at lepton colliders show that coarse bounds on $\lambda_{4}$ could be obtained and would complement the information from triple Higgs production, improving the ultimate results via a combination. At variance with the case of $\lambda_{3}, \lambda_{4}$-dependent loop corrections are ultraviolet (UV) divergent and in order to be renormalised they have to be performed in an Effective-Field-Theory (EFT) framework. The renormalisation procedure and the relevant counterterms have been provided in ref. [68]. This framework has to be used also when the interest is focused only on independent variations of $\lambda_{3}$ and $\lambda_{4}$, so that UV-finite results can be obtained.

The similar calculation for the case of hadronic collisions is computationally more involved, since the process $p p \rightarrow H H$ involves the loop-induced $g g \rightarrow H H$ partonic process at Born level and therefore the sensitivity on $\lambda_{4}$ originates from two-loop amplitudes. The first incomplete estimation of these effects has been presented in ref. [72], showing the possibility of following this strategy also at future hadron colliders.

In this paper we analyse this strategy in detail and provide the first complete and consistent computation of the relevant contributions to $g g \rightarrow H H$ at two loops. All the two-loop diagrams involving $\lambda_{4}$ are taken into account and numerically evaluated via PYSECDEC $[73,74]$ without any further approximation. Moreover, following the approach of ref. [68], we take into account also corrections induced by additional $\lambda_{3}$ effects at two loops, which are non negligible for large values of $\lambda_{3}$, and we renormalise the ensuing UV divergences. We perform this calculation at the differential level and we consider the $b \bar{b} \gamma \gamma$ signature emerging from the decays of the Higgs bosons as a first application. This channel has been identified as the most promising one [21, 36, 37, 56, 75-77] and it allows for the reconstruction of the di-Higgs invariance mass $m(H H)$. Following the analyses in ref. [21], we study the constraints that can be set on $\lambda_{3}$ and $\lambda_{4}$ via the measurement of the $m(H H)$ distribution from $b \bar{b} \gamma \gamma$ events for two different experimental setups: the LHC with $3000 \mathrm{fb}^{-1}$ integrated luminosity (HL-LHC) and at a $100 \mathrm{TeV}$ collider with $30 \mathrm{ab}^{-1}$ integrated luminosity. The EFT parametrisation allows us to consider both the generic case, where $\lambda_{3}$ and $\lambda_{4}$ can vary independently, and a "well-behaved" EFT approach, where higher dimension operators induce smaller effects and thus $\lambda_{4}$ depends on $\lambda_{3}$. In both cases we assume that the dominant BSM effects originate from the distortion of the Higgs potential, namely, anomalous interactions of the Higgs boson with other SM particles lead to subdominant effects. This approach is adequate to establish the sensitivity. 
The paper is organised as follows. We first provide details on the computational framework, clarifying the theoretical assumptions, identifying the most relevant terms and describing the most important elements and features of the two-loop computation in section 2. Section 3 presents the results of the computation at the total as well as differential level, while in section 4 constraints that can be derived from future measurements at the LHC and at $100 \mathrm{TeV}$ FCC are discussed in two different scenarios. We summarise our findings in section 5. Three appendices contain complementary and technical information.

\section{Calculation}

\subsection{Parametrisation of $\lambda_{3}$ and $\lambda_{4}$ effects}

As already mentioned in the introduction, in order to vary the cubic and quartic Higgs self couplings $\lambda_{3}$ and $\lambda_{4}$ independently at all orders in perturbation theory in a consistent way, an EFT approach where operators are defined above the EWSB scale and respect all symmetries, hidden or not, has to be employed. This allows one to systematically identify gauge invariant and UV finite subsets of diagrams. For this reason, we will use the computational framework introduced and described in detail in ref. [68]. In this section we summarise the most important aspects and we highlight some differences w.r.t. ref. [68].

Starting from the SM Higgs potential

$$
V^{\mathrm{SM}}(\Phi)=-\mu^{2}\left(\Phi^{\dagger} \Phi\right)+\lambda\left(\Phi^{\dagger} \Phi\right)^{2}
$$

we denote NP effects as $V^{\mathrm{NP}}$ so that the general form of the potential can be written as

$$
V(\Phi)=V^{\mathrm{SM}}(\Phi)+V^{\mathrm{NP}}(\Phi), \quad \Phi=\left(\begin{array}{c}
G^{+} \\
\frac{1}{\sqrt{2}}\left(v+H+i G^{0}\right)
\end{array}\right)
$$

where the symbol $\Phi$ refers to the Higgs doublet. Using the conventions of ref. [78], the most general form of an $\mathrm{SU}(2)$-invariant $V^{\mathrm{NP}}$ potential reads

$$
V^{\mathrm{NP}}(\Phi) \equiv \sum_{n=3}^{\infty} \frac{c_{2 n}}{\Lambda^{2 n-4}}\left(\Phi^{\dagger} \Phi-\frac{1}{2} v^{2}\right)^{n} .
$$

One of the advantages of this parameterisation is that at tree-level $\lambda_{3}$ only depends on $c_{6}$ and $\lambda_{4}$ only on $c_{6}$ and $c_{8}$. Indeed, after EWSB, we can rewrite $V(\Phi)$ as

$$
V(H)=\frac{1}{2} m_{H}^{2} H^{2}+\lambda_{3} v H^{3}+\frac{1}{4} \lambda_{4} H^{4}+\lambda_{5} \frac{H^{5}}{v}+O\left(H^{6}\right),
$$

and thus define the self couplings $\lambda_{3}$ and $\lambda_{4}$ via

$$
\begin{gathered}
\kappa_{3} \equiv \frac{\lambda_{3}}{\lambda_{3}^{\mathrm{SM}}}=1+\frac{c_{6} v^{2}}{\lambda \Lambda^{2}} \equiv 1+\bar{c}_{6}, \\
\kappa_{4} \equiv \frac{\lambda_{4}}{\lambda_{4}^{\mathrm{SM}}}=1+\frac{6 c_{6} v^{2}}{\lambda \Lambda^{2}}+\frac{4 c_{8} v^{4}}{\lambda \Lambda^{4}} \equiv 1+6 \bar{c}_{6}+\bar{c}_{8} .
\end{gathered}
$$



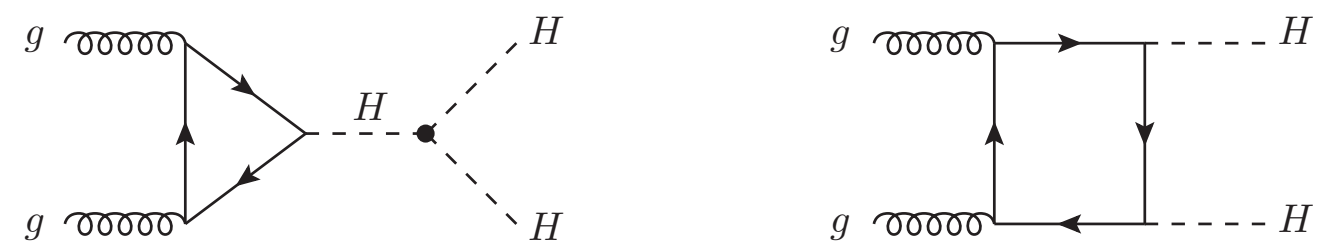

Figure 1. Double Higgs production at LO in SM. The triangle diagram is sensitive to the cubic coupling.

The quantities $\lambda_{3}^{\mathrm{SM}}$ and $\lambda_{4}^{\mathrm{SM}}$ are the values of $\lambda_{3}$ and $\lambda_{4}$ in the SM, respectively, and read

$$
\lambda_{3}^{\mathrm{SM}}=\lambda_{4}^{\mathrm{SM}}=\lambda=\frac{m_{H}^{2}}{2 v^{2}} .
$$

In other words, the barred quantities $\bar{c}_{6}$ and $\bar{c}_{8}$ are simply $c_{6}$ and $c_{8}$ normalised in such a way that relations to $\kappa_{3}$ and $\kappa_{4}$ are simple. In particular

$$
\begin{aligned}
& \bar{c}_{6} \equiv \frac{c_{6} v^{2}}{\lambda \Lambda^{2}}=\kappa_{3}-1, \\
& \bar{c}_{8} \equiv \frac{4 c_{8} v^{4}}{\lambda \Lambda^{4}}=\kappa_{4}-1-6\left(\kappa_{3}-1\right) .
\end{aligned}
$$

Using the parameterisation in eq. (2.3) and eqs. (2.5) and (2.6), or equivalently eqs. (2.8) and (2.9), we can trade $\kappa_{3}$ and $\kappa_{4}$ with only two other parameters, $\bar{c}_{6}$ and $\bar{c}_{8}$. In so doing, we can always think of using the EFT approach as a way to obtain gauge invariant and UV-finite results in the anomalous coupling approach. ${ }^{1}$ We note that, $a$ priori, in a well-behaved EFT higher dimensional effects are expected to suppressed by a large scale $\Lambda$. Thus, in the first approximation, deviations in $\kappa_{3}$ and $\kappa_{4}$ are strongly correlated, i.e., $\left(\kappa_{4}-1\right) \simeq 6\left(\kappa_{3}-1\right)$, see also eq. (2.9). Similarly to what as been done in refs. $[68,71,72]$, in this work we adopt as starting point an agnostic attitude towards the values that $\kappa_{3}$ and $\kappa_{4}$ can assume, in order to cover the sensitivity that future colliders can probe. We will later comment on bounds on $\kappa_{3}$ and $\kappa_{4}$ making different assumptions.

In this work we calculate the effects of anomalous cubic and quartic couplings in double Higgs production at hadron colliders. While $\lambda_{3}$ affects the $g g \rightarrow H H$ amplitude already at the Born level, $\lambda_{4}$ enters only via NLO EW corrections, i.e., at the two-loop level. Before discussing the details of the calculation it is convenient to anticipate what are the quantities that enter in our phenomenological predictions. In figure 1 we display the one-loop diagrams of the Born amplitude in $H H$ production. While the triangle (left diagram) depends on $\lambda_{3}$, the box (right diagram) does not. Moreover, it is well known that the interference effects between the two diagrams leads to large cancellations. As already mentioned, higher-order QCD corrections are large and therefore cannot be neglected. However, in this work we will assume that they factorise from the two-loop EW effects that we calculate. While the accuracy of this assumption has been directly tested only in very few cases [79-81], it has been often employed in the past, both due to the difficulty of

\footnotetext{
${ }^{1}$ Note that using the alternative parameterisation $V^{\mathrm{NP}}(\Phi) \equiv \sum_{n=3}^{\infty} \frac{c_{2 n}^{\prime}}{\Lambda^{2 n}-4}\left(\Phi^{\dagger} \Phi\right)^{n}$ both $\lambda_{3}$ and $\lambda_{4}$ would depend on all the $c_{i}^{\prime}$ coefficients already at the tree level.
} 
calculating QCD-EW mixed corrections and due to the theoretical arguments supporting its validity. Two-loop corrections to $H H$ production involve further $\lambda_{3}$ effects and introduce a $\lambda_{4}$ dependence, as can be seen in figure 2. All the contributions arising from the two-loop topologies depicted in figure 2 have been evaluated and renormalised via UV counterterms; more details concerning the calculation are given in section 2.2 .

Following the approach presented in ref. [68] for $e^{+} e^{-}$collisions, we define the quantity to be used in phenomenological investigations as

$$
\sigma_{\mathrm{NLO}}^{\mathrm{pheno}}=\sigma_{\mathrm{LO}}+\Delta \sigma_{\bar{c}_{6}}+\Delta \sigma_{\bar{c}_{8}}
$$

where

$$
\sigma_{\mathrm{LO}}=\sigma_{0}+\sigma_{1} \bar{c}_{6}+\sigma_{2} \bar{c}_{6}^{2},
$$

is the LO prediction. In eq. (2.11), $\sigma_{0}$ is the SM prediction, $\sigma_{1}$ corresponds to the leading contribution in the EFT expansion, being of order $(v / \Lambda)^{2}$, while $\sigma_{2}$ is of order $(v / \Lambda)^{4}$ and corresponds to the squared EFT term. Clearly, no contribution proportional to $\bar{c}_{8}$ appears at LO. The NLO corrections are included through the terms

$$
\begin{aligned}
\Delta \sigma_{\bar{c}_{6}} & =\bar{c}_{6}^{2}\left[\sigma_{30} \bar{c}_{6}+\sigma_{40} \bar{c}_{6}^{2}\right]+\tilde{\sigma}_{20} \bar{c}_{6}^{2}, \\
\Delta \sigma_{\bar{c}_{8}} & =\bar{c}_{8}\left[\sigma_{01}+\sigma_{11} \bar{c}_{6}+\sigma_{21} \bar{c}_{6}^{2}\right],
\end{aligned}
$$

which are the loop corrections induced by $\bar{c}_{6}$ on top of $\bar{c}_{6}$ and the two-loop $\bar{c}_{8}$-dependent part, respectively. They both originate from the topologies shown in figure 2 . In the following we explain the rationale behind these formulae and the meaning of the different $\sigma_{i(j)} \bar{c}_{6}^{i} \bar{c}_{8}^{j}$ terms entering them.

First of all it is important to note that we organise the different contributions in terms of $\bar{c}_{6}$ and $\bar{c}_{8}$ and not $\lambda_{3}$ and $\lambda_{4}$. As explained in ref. [68] this organisation reflects the necessary EFT expansion that has to be performed in order to renormalise UV divergences and obtain gauge invariant predictions. We recall that $\bar{c}_{6}$ can be directly related to $\lambda_{3}$, while $\bar{c}_{8}$ captures the violation of the relation $\kappa_{4}=6 \kappa_{3}-5$, which holds if only $\bar{c}_{6}$ is present, cf. eqs. (2.8) and (2.9).

Our goal is not to determine the ultimate precision that can be achieved at future colliders on $\bar{c}_{6}$ and $\bar{c}_{8}$. Rather, we want to perform the first sensibility study on the determination of the cubic and quartic Higgs self couplings via double Higgs production at future hadron colliders. For this reason, SM EW corrections on top of $\sigma_{\mathrm{LO}}$ are not taken into account. Since we are agnostic about the possible size of $\bar{c}_{6}$, large cubic couplings are possible and lead to sizable enhancements via topologies such as $(d)$ in figure $2[68,82]$. For this reason, in $\Delta \sigma_{\bar{c}_{6}}$ we take into account all the contributions of order $\bar{c}_{6}^{3}$ and $\bar{c}_{6}^{4}$. These two contributions are relevant only for large $\bar{c}_{6}$, since otherwise they are suppressed w.r.t. the contributions appearing at LO. We remind that in refs. $[68,82]$ it has been shown that $\Delta \sigma_{\bar{c}_{6}}$, and therefore $\sigma_{\mathrm{NLO}}^{\text {pheno }}$, in general makes sense only in the range $\left|\bar{c}_{6}\right|<5$. Outside this range perturbativity is violated for any prediction involving the bulk of $H H$ production. We will comment more on this point in section 3. At variance with ref. [68], we include also 

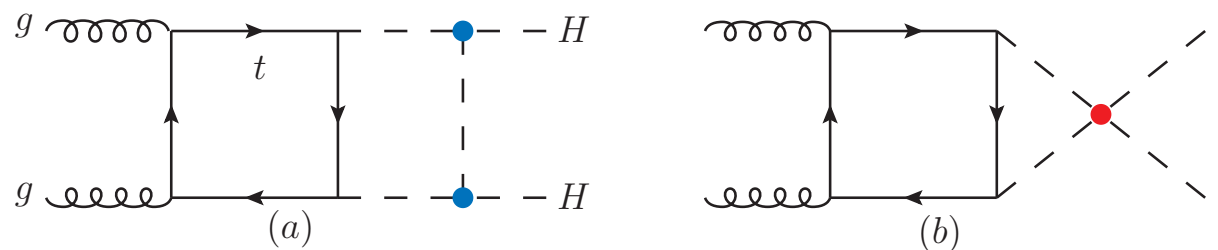

(b)



(c)

(d)
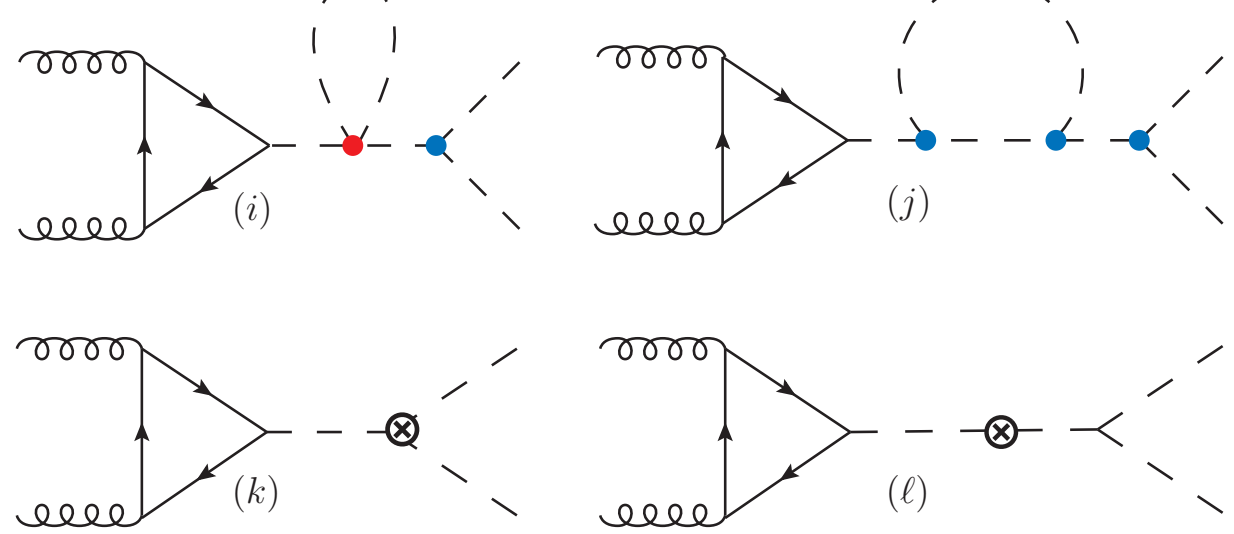

Figure 2. Two-loop topologies involving $\bar{c}_{6}$ and $\bar{c}_{8}$ effects on Higgs self coupling in $g g \rightarrow H H$. Except diagrams $(g)$ and $(h)$, all topologies are present in the SM. We have marked with a blob all the vertices involving $\bar{c}_{6}$ and $\bar{c}_{8}$; cubic vertices are in blue while quartic ones are in red. Diagrams $(a)$ $(c)$ are non-factorisable two-loop topologies. Diagrams $(d)-(h)$, together with the counterterm $(k)$, can be evaluated via the one-loop form factor $V[H H H]$, while $(i),(j)$ and $(l)$ with the $P[H H]$ one. 
the term $\tilde{\sigma}_{20} \bar{c}_{6}^{2}$ in eq. (2.12). This term includes only part of the two-loop contributions of order $\bar{c}_{6}^{2}$ and its purpose is to preserve the large cancellations that, similarly to the case of $\sigma_{\mathrm{LO}}$, are present in $\Delta \sigma_{\bar{c}_{6}}$ and especially are distributed among different $\sigma_{i 0}$ terms. On the other hand, it is relevant only for $\bar{c}_{6} \sim 2$ where the cross section reaches the smallest value and the cancellations are the largest. ${ }^{2}$

The quantity $\Delta \sigma_{\bar{c}_{8}}$ is the most relevant part of our computation and it solely induces the sensitivity on $\bar{c}_{8}$. At variance with ref. [72], where only the topology $(b)$ has been considered, in this term we take into account also all the contributions originating from topologies $(e)-(i)$, which contribute at the same level and therefore cannot be ignored in any gauge-invariant calculation. ${ }^{3}$ Also for the case of $\bar{c}_{8}$, a theoretical bound based on the perturbativity requirement can be set [68] and corresponds to $\left|\bar{c}_{8}\right|<31$.

\subsection{Organisation of the calculation}

In this section we give more details about our computational framework. Let us first consider the origin of the contributions in eqs. (2.12) and (2.13), in particular the presence of the term $\tilde{\sigma}_{20}$. Using the same notations as for the $\sigma_{i(j)}$ terms, we define the different contributions of order $\bar{c}_{6}^{i} \bar{c}_{8}^{j}$ entering the $\mathcal{M}(g g \rightarrow H H)$ amplitude as $\mathcal{M}_{i(j)}$. Denoting by $\mathcal{M}^{1 \mathrm{~L}}$ and $\mathcal{M}^{2 \mathrm{~L}}$ the one-loop and two-loop amplitudes, we define

$$
\begin{aligned}
& \mathcal{M}^{1 \mathrm{~L}}=\mathcal{M}_{0}^{1 \mathrm{~L}}+\bar{c}_{6} \mathcal{M}_{1}^{1 \mathrm{~L}}, \\
& \mathcal{M}^{2 \mathrm{~L}}=\sum_{i+2 j \leq 3} \bar{c}_{6}^{i} \bar{c}_{8}^{j} \mathcal{M}_{i j}^{2 \mathrm{~L}} .
\end{aligned}
$$

The SM term $\mathcal{M}_{0}^{1 \mathrm{~L}}$ receives contributions from both the one-loop triangle and box diagrams in figure 1. The relation between eqs. (2.12) and (2.13) and the $\mathcal{M}_{i(j)}$ terms is:

$$
\begin{aligned}
& \Delta \sigma_{\bar{c}_{6}} \propto 2 \Re\left[\left(\mathcal{M}_{0}^{1 \mathrm{~L}}+\bar{c}_{6} \mathcal{M}_{1}^{1 \mathrm{~L}}\right)\left(\bar{c}_{6}^{2} \mathcal{M}_{20}^{2 \mathrm{~L}}+\bar{c}_{6}^{3} \mathcal{M}_{30}^{2 \mathrm{~L}}\right)^{*}\right], \\
& \Delta \sigma_{\bar{c}_{8}} \propto 2 \Re\left[\left(\mathcal{M}_{0}^{1 \mathrm{~L}}+\bar{c}_{6} \mathcal{M}_{1}^{1 \mathrm{~L}}\right)\left(\bar{c}_{8} \mathcal{M}_{01}^{2 \mathrm{~L}}+\bar{c}_{6} \bar{c}_{8} \mathcal{M}_{11}^{2 \mathrm{~L}}\right)^{*}\right] .
\end{aligned}
$$

In other words, $\Delta \sigma_{\bar{c}_{6}}$ and $\Delta \sigma_{\bar{c}_{8}}$ originate from the interference of $\mathcal{M}^{1 \mathrm{~L}}$ with the terms with the largest dependence on $\bar{c}_{6},\left(\bar{c}_{6}^{2} \mathcal{M}_{20}^{2 \mathrm{~L}}+\bar{c}_{6}^{3} \mathcal{M}_{30}^{2 \mathrm{~L}}\right)$, and all the terms that depend on $\bar{c}_{8}$, $\left(\bar{c}_{8} \mathcal{M}_{01}^{2 \mathrm{~L}}+\bar{c}_{6} \bar{c}_{8} \mathcal{M}_{11}^{2 \mathrm{~L}}\right)$. However, while the perturbative orders in $\Delta \sigma_{\bar{c}_{8}}$ and the interference terms emerging from the r.h.s. of eq. (2.17) are in one-to-one correspondence, this is not true for $\Delta \sigma_{\bar{c}_{6}}$. The term $2 \Re\left[\left(\mathcal{M}_{0}^{1 \mathrm{~L}}\right)\left(\mathcal{M}_{20}^{2 \mathrm{~L}}\right)^{*}\right]$ from the r.h.s. of eq. $(2.16)$, which gives rise to $\tilde{\sigma}_{20}$, multiplies the same $\bar{c}_{6}$ powers as the term $2 \Re\left[\left(\mathcal{M}_{1}^{1 \mathrm{~L}}\right)\left(\mathcal{M}_{10}^{2 \mathrm{~L}}\right)^{*}\right]$, which we do not include in our computation. As already mentioned, we include the (formally subleading) term $\tilde{\sigma}_{20}$ because of the large cancellations among the triangle and box topologies at LO, and the fact that they contribute to different $\bar{c}_{6}$ powers; these cancellations are not substantially

\footnotetext{
${ }^{2}$ We have verified that the inclusion of the analogous term in the $e^{+} e^{-}$studies in ref. [68] would lead to negligible differences.

${ }^{3}$ Note that the topology $(g)$ involves a $H^{5}$ interaction which in principle depends also on the $\bar{c}_{10}$ Wilson coefficient form the dimension-10 operator $\left(\Phi^{\dagger} \Phi-\frac{1}{2} v^{2}\right)^{5}$. As discussed in ref. [68], the effect of this diagram can be redefined as a constant shift on $\bar{c}_{6}$ and therefore our calculation is sensitive on a linear combination of $\bar{c}_{6}$ and $\bar{c}_{10}$. Thus we set $\bar{c}_{10}$ equal to zero. Nevertheless, the $\bar{c}_{6}$ and $\bar{c}_{8}$ contributions emerging from this diagrams are taken into account. See ref. [68] for more details.
} 
spoiled by NLO corrections. By keeping at the same level the entire $\mathcal{M}^{1 \mathrm{~L}}$ amplitude of eq. (2.14) in the interference leading to $\Delta \sigma_{\bar{c}_{6}}$ we avoid that similar cancellations in NLO corrections are truncated by the $\bar{c}_{6}$ expansion. As already mentioned, this is relevant only for $\bar{c}_{6} \sim 2$, where the total cross section has the minimum value, precisely due to the aforementioned cancellations. We remark, however, that this does not change the formal accuracy of our NLO corrections, which is of order $\bar{c}_{6}^{3}$ and $\bar{c}_{6}^{4}$.

The two-loop contributions entering the different $\mathcal{M}_{i j}$ sub-amplitudes can be further classified into three types:

- Factorisable two-loop contributions $(\mathcal{F})$,

- Non-factorisable two-loop contributions $(\mathcal{N})$,

- Higgs wave-function counterterms $(\mathcal{W})$.

This classification is based on Feynman diagrams and can be easily understood from the topologies in figure 2. The first category $\mathcal{F}$ corresponds to the factorisable topologies $(d)$ $(j)$, together with the vertex counterterms in topologies $(k)$ and $(l)$. Their contributions are separately UV divergent, but their sum is finite, also for each separate $\bar{c}_{6}^{i} \bar{c}_{8}^{j}$ order considered in this work. In particular, the topologies $(i),(j)$ and $(l)$ can be evaluated together via the UV-finite $P[H H]$ form factor given in ref. [68], while all the remaining topologies from category $\mathcal{F}$ via the UV-finite $V[H H H]$ form factor given in the same reference. We remind the reader that topology $(d)$ is $\mathrm{UV}$ finite.

The non-factorisable two-loop contributions correspond to the topologies $(a)-(c)$ which are not available. From a technical point of view, their computation is the most difficult and important part of this work. Details are given in section 2.3. Moreover, we find that numerically their phenomenological impact is non-negligible w.r.t. the factorisable ones.

We remind the reader that the Higgs wave-function renormalisation constant involves a quadratic dependence on $\kappa_{3}$ and therefore both a quadratic and linear dependence on $\bar{c}_{6}[61,62]$. Moreover, its contribution is UV-finite. Following the conventions of ref. [68], its contribution is not included in the $P[H H]$ and $V[H H H]$ form factors and has to be separately added. The third category $\mathcal{W}$ corresponds to these additional contributions, which can be easily calculated via the LO diagrams and the SM contribution of $\lambda_{3}$ to the Higgs wave-function, namely,

$$
\delta Z_{H}^{\mathrm{SM}, \lambda_{3}}=-\frac{9 \lambda m_{H}^{2}}{16 \pi^{2}} B_{0}^{\prime}\left(m_{H}^{2}, m_{H}^{2}, m_{H}^{2}\right),
$$

where $B_{0}^{\prime}\left(m_{H}^{2}, m_{H}^{2}, m_{H}^{2}\right)$ is the derivative of the $B_{0}\left(p^{2}, m_{H}^{2}, m_{H}^{2}\right)$ scalar integral evaluated at $p^{2}=m_{H}^{2}$.

Based on the classifications we have just introduced, the different $\mathcal{M}_{i j}^{2 \mathrm{~L}}$ terms can be further divided into

$$
\begin{array}{ll}
\mathcal{M}_{20}^{2 \mathrm{~L}}=\mathcal{M}_{20}^{\mathcal{W}}+\mathcal{M}_{20}^{\mathcal{F}}+\mathcal{M}_{20}^{\mathcal{N}}, \\
\mathcal{M}_{30}^{2 \mathrm{~L}}=\mathcal{M}_{30}^{\mathcal{W}}+\mathcal{M}_{30}^{\mathcal{F}}, \\
\mathcal{M}_{01}^{2 \mathrm{~L}}= & \mathcal{M}_{01}^{\mathcal{F}}+\mathcal{M}_{01}^{\mathcal{N}}, \\
\mathcal{M}_{11}^{2 \mathrm{~L}}= & \mathcal{M}_{11}^{\mathcal{F}} .
\end{array}
$$


Moreover, it is useful to further divide the amplitude into its spin components. In view of the description of the calculation of two-loop non-factorisable diagrams, it is important to note that only the topology $(a)$ includes both a spin- 0 and spin- 2 component; all the other topologies in figure 2 are solely spin- 0 . In the case of one-loop diagrams, the triangle is also solely spin- 0 , while the box includes both a spin- 0 and spin- 2 component. Thus, the spin-2 contribution of the box diagram interferes only with the spin-2 component of the topology $(a)$, while the spin- 0 part of the box diagram and the triangle diagram interfere with all the two-loop topologies.

Since the diagrams in the topology $(a)$, which involves both spin-0 and spin-2 components, lead to contributions of order $\bar{c}_{6}^{2}$, we can further define

$$
\begin{aligned}
& \mathcal{M}_{0,20}=\mathcal{M}_{0,20}^{\mathcal{W}}+\mathcal{M}_{0,20}^{\mathcal{F}}+\mathcal{M}_{0,20}^{\mathcal{N}}, \\
& \mathcal{M}_{2,20}=\mathcal{M}_{2,20}^{\mathcal{W}}+\mathcal{M}_{2,20}^{\mathcal{N}}
\end{aligned}
$$

where the first lower index denotes the spin component. With this notation we can directly express the $\mathcal{M}_{0,20}^{\mathcal{W}}, \mathcal{M}_{2,20}^{\mathcal{W}}$ and also $\mathcal{M}_{30}^{\mathcal{W}}$ terms as

$$
\begin{aligned}
& \mathcal{M}_{0,20}^{\mathcal{W}}= \delta Z_{H}^{\mathrm{SM}, \lambda_{3}}\left(2 \mathcal{M}_{0,1}^{1 \mathrm{~L}}+\mathcal{M}_{0,0}^{1 \mathrm{~L}}\right), \\
& \mathcal{M}_{2,20}^{\mathcal{W}}=\delta Z_{H}^{\mathrm{SM}, \lambda_{3}} \mathcal{M}_{2,0}^{1 \mathrm{~L}}, \\
& \mathcal{M}_{30}^{\mathcal{W}}=\mathcal{M}_{0,30}^{\mathcal{W}}=\delta Z_{H}^{\mathrm{SM}, \lambda_{3}} \mathcal{M}_{0,1}^{1 \mathrm{~L}} .
\end{aligned}
$$

Therefore, thanks to eqs. (2.21)-(2.23) and the formulae for the $P[H H]$ and $V[H H H]$ form factors provided in ref. [68], both the $\mathcal{F}$ and $\mathcal{W}$ contributions can be calculated. The only missing component in our calculation are the non-factorizable $(\mathcal{N})$ contributions, which are discussed in the next section.

\subsection{Two-loop non-factorisable terms}

\subsubsection{Reduction to form factors}

All the non-factorisable $(\mathcal{N})$ contributions originate from the topologies $(a),(b)$ and $(c)$ in figure 2, these topologies can be further divided in sub-topologies; we show them for $(a)$ and $(b)$ in figure 3 , those for $(c)$ can be trivially obtained adding an $H$ propagator in $(b)$. In the topology $(a)$ (double-box) there are in total 6 diagrams of which 3 are independent due to charge conjugation property of the fermion loop: 2 planar, $\left(a_{1}\right)$ and $\left(a_{2}\right)$, and 1 nonplanar, $\left(a_{2}\right)$. In the topology $(b)$ (box-triangle) there are in total 3 diagrams, 2 of them are planar and charged conjugate, leading to $\left(b_{1}\right)$, the remaining diagram is instead non-planar, $\left(b_{2}\right)$. The case of $(c)$, is analogous to $(b)$, including an $H$ propagator. Topology $(a)$, as we already said, contributes to both $\mathcal{M}_{0,20}^{\mathcal{N}}$ and $\mathcal{M}_{2,20}^{\mathcal{N}}$, while topology $(c)$ is in one-to-one correspondence with $\mathcal{M}_{01}^{\mathcal{N}}$. Topology (b) contributes also to $\mathcal{M}_{0,20}^{\mathcal{N}}$, which is therefore the only non-factorisable term receiving contributions from two different topologies. We can 

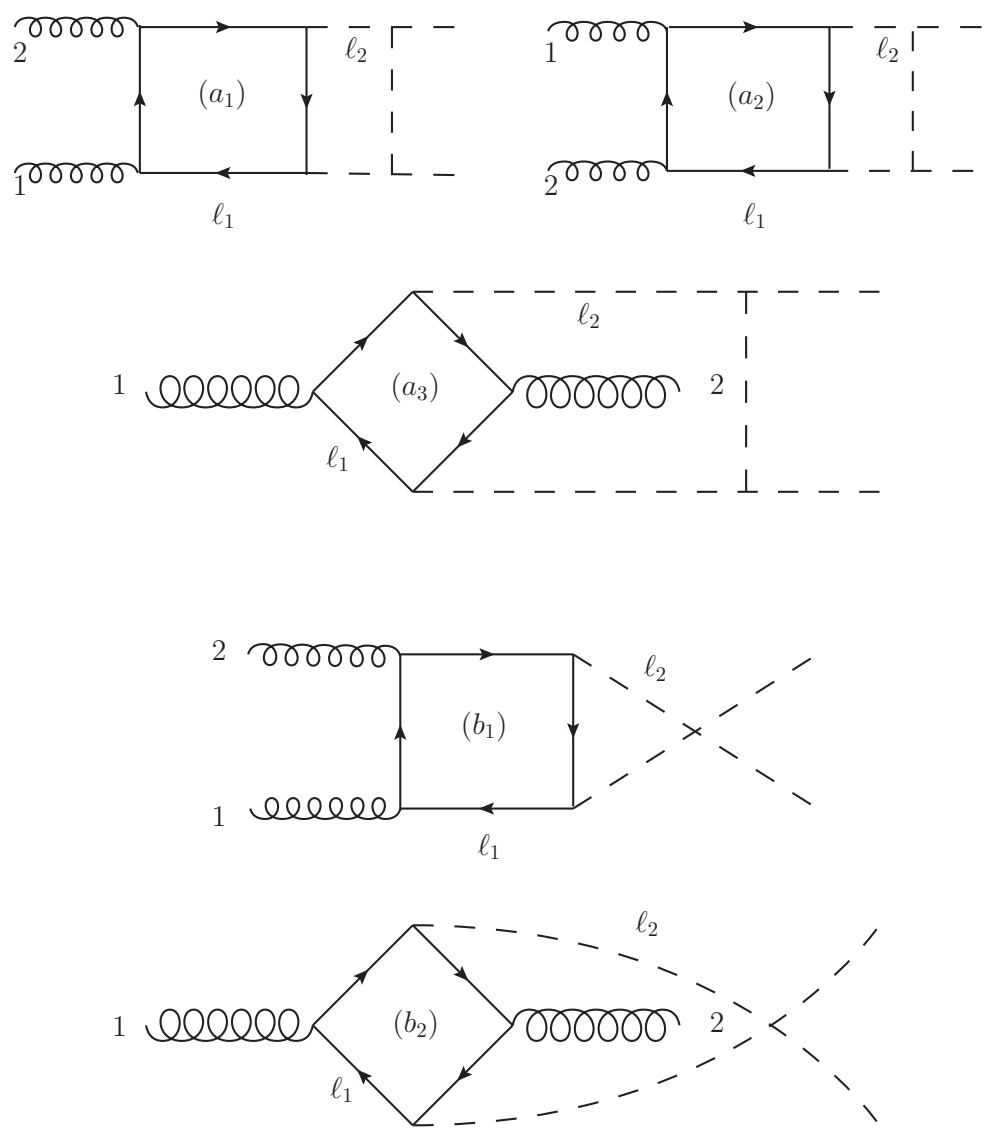

Figure 3. Non-factorizable two-loop diagrams of classes $(a)$ and $(b)$.

schematically summarise all this information as

$$
\begin{aligned}
& \mathcal{M}_{2,20}^{\mathcal{N}}, \mathcal{M}_{0,20}^{\mathcal{N}} \Longleftarrow \mathcal{M}_{a}=2\left(\mathcal{M}_{a_{1}}+\mathcal{M}_{a_{2}}+\mathcal{M}_{a_{3}}\right), \\
& \mathcal{M}_{0,01}^{\mathcal{N}}=\mathcal{M}_{01}^{\mathcal{N}} \Longleftarrow \mathcal{M}_{b}=2 \mathcal{M}_{b_{1}}+\mathcal{M}_{b_{2}}, \\
& \mathcal{M}_{0,20}^{\mathcal{N}} \Longleftarrow \mathcal{M}_{c}=\mathcal{M}_{b} \times \frac{6 v^{2}}{\lambda_{4}} \frac{\lambda_{3}^{2}}{s-m_{H}^{2}},
\end{aligned}
$$

where $\sqrt{s}$ is the invariant mass of di-Higgs bosons, and the $\Longleftarrow$ arrow should be understood as "contributes to" and we have further remarked that $\mathcal{M}_{01}^{\mathcal{N}}$ is all spin-zero. It is important to note that the sums of diagrams in each topology $(a),(b)$ and $(c)$ are separately finite and gauge invariant.

The calculation of all the non-factorisable two-loop diagrams is performed via numerical methods. As a first step, two-loop diagrams are generated with QGRAF [83] and the amplitudes are written in FORM [84] in $d=4-2 \epsilon$ dimensions. Then, the amplitudes are projected onto spin-0 and spin-2 form factors. ${ }^{4}$ Assigning the following on-shell $p_{i}$ momenta to the external particles,

$$
g\left(p_{1}\right)+g\left(p_{2}\right) \rightarrow H\left(-p_{3}\right)+H\left(-p_{4}\right),
$$

\footnotetext{
${ }^{4}$ In this work, this projection has been used also for the evaluation of $\mathcal{F}$ and $\mathcal{W}$ contributions.
} 
where all the $p_{i}$ are considered as incoming, both $\mathcal{M}^{1 \mathrm{~L}}$ and $\mathcal{M}^{2 \mathrm{~L}}$, and any of their gauge-invariant sub-amplitudes, can be projected onto two spin-0 and spin-2 bases $\mathcal{A}_{0}$ and $\mathcal{A}_{2}[41,85]$, and expressed via corresponding form factors denoted as $F_{0}$ and $F_{2}$. Specifically,

$$
\mathcal{M}^{\mu_{1} \mu_{2}} \epsilon_{1, \mu_{1}} \epsilon_{2, \mu_{2}}=\delta^{c_{1} c_{2}} \mathcal{A}_{0}^{\mu_{1} \mu_{2}} \epsilon_{1, \mu_{1}} \epsilon_{2, \mu_{2}} F_{0}+\delta^{c_{1} c_{2}} \mathcal{A}_{2}^{\mu_{1} \mu_{2}} \epsilon_{1, \mu_{1}} \epsilon_{2, \mu_{2}} F_{2} .
$$

In eq. (2.28) $\epsilon_{1}$ and $\epsilon_{2}$ are the (transverse) polarisation vectors for the two incoming on-shell gluons, while $\mu_{1}$ and $\mu_{2}\left(c_{1}\right.$ and $\left.c_{2}\right)$ are their corresponding Lorentz(colour) indices. We choose to normalize the tensor bases $\mathcal{A}_{0}^{\mu_{1} \mu_{2}}$ and $\mathcal{A}_{2}^{\mu_{1} \mu_{2}}$ according to the following conditions ${ }^{5}$

$$
\mathcal{A}_{0} \cdot \mathcal{A}_{0}=\mathcal{A}_{2} \cdot \mathcal{A}_{2}=2, \mathcal{A}_{0} \cdot \mathcal{A}_{2}=0 \text {. }
$$

Explicitly, they $\operatorname{read}^{6}$

$$
\begin{aligned}
\mathcal{A}_{0}^{\mu_{1} \mu_{2}}= & \sqrt{\frac{2}{d-2}}\left(g^{\mu_{1} \mu_{2}}-\frac{p_{1}^{\mu_{2}} p_{2}^{\mu_{1}}}{p_{1} \cdot p_{2}}\right) \\
\mathcal{A}_{2}^{\mu_{1} \mu_{2}}= & \sqrt{\frac{d-2}{2(d-3)}}\left(-\frac{d-4}{d-2}\left[g^{\mu_{1} \mu_{2}}-\frac{p_{1}^{\mu_{2}} p_{2}^{\mu_{1}}}{p_{1} \cdot p_{2}}\right]+g^{\mu_{1} \mu_{2}}\right. \\
& \left.+\frac{\left(p_{3} \cdot p_{3}\right) p_{1}^{\mu_{2}} p_{2}^{\mu_{1}}+\left(2 p_{1} \cdot p_{2}\right) p_{3}^{\mu_{1}} p_{3}^{\mu_{2}}-\left(2 p_{1} \cdot p_{3}\right) p_{2}^{\mu_{1}} p_{3}^{\mu_{2}}-\left(2 p_{2} \cdot p_{3}\right) p_{3}^{\mu_{1}} p_{1}^{\mu_{2}}}{p_{T}^{2}\left(p_{1} \cdot p_{2}\right)}\right),
\end{aligned}
$$

where $p_{T}^{2}=\left(s_{13} s_{23}-m_{H}^{4}\right) / s_{12}$ denotes the square of the Higgs-boson transverse momentum w.r.t. the gluons in the center-of-mass rest frame, using the convention $s_{i j}=\left(p_{i}+p_{j}\right)^{2}$.

After the above projection, we obtain the spin-dependent non-factorisable amplitudes $\mathcal{M}_{0,20}^{\mathcal{N}}, \mathcal{M}_{2,20}^{\mathcal{N}}$ and $\mathcal{M}_{0,01}^{\mathcal{N}}$ written in terms of form factors, i.e., $F_{0,20}^{\mathcal{N}}, F_{2,20}^{\mathcal{N}}$ and $F_{0,01}^{\mathcal{N}}$ where

$$
F_{0,20}^{\mathcal{N}}=F_{0, a}+F_{0, c}, \quad F_{2,20}^{\mathcal{N}}=F_{2, a}, \quad \text { and } \quad F_{0,01}^{\mathcal{N}}=F_{0, b} .
$$

In other words, all the $\mathcal{N}$ contributions entering our calculation can be expressed via the $F_{0,20}^{\mathcal{N}}, F_{2,20}^{\mathcal{N}}$ and $F_{0,01}^{\mathcal{N}}$ form factors, which in turn depend on the non-vanishing spin-0 and spin-2 projections of the $(a)-(c)$ topologies, $F_{0, a}, F_{0, b}, F_{0, c}$ and $F_{2, a}$.

\subsubsection{Numerical evaluation of the form factors}

The form factors $F_{0, a}, F_{0, b}, F_{0, c}$ and $F_{2, a}$ are computed with PySecDec [73, 74], a toolbox for the numerical evaluation of multi-loop integrals. We remind the reader that PYSECDEC can readily compute loop integrals with massive internal lines and/or off-shell legs. Moreover, compared to its predecessor SECDEC 3 [86], it facilitates the creation of integral libraries, allowing for a direct incorporation of the code into the calculation of the full amplitude.

Before using PySECDEC, we simplify the numerators of the loop integrals in the form factors, in order to obtain tensor integrals that optimise the speed of the computation. It is

\footnotetext{
${ }^{5}$ The inner product stands for the contraction with polarisation vectors and summation over all physical polarisations.

${ }^{6}$ The expression for the second projector in ref. [85] contains a typo that is corrected here.
} 
important to note that the form factors $F_{0, a}$ and $F_{2, a}$ involve 7-propagator diagrams while $F_{0, b}$ and $F_{0, c} 6$-propagator ones. Using propagator identities in FORM, we obtain a total of 11 integral expressions for $F_{0, a}, 24$ for $F_{2, a}$ and 9 for $F_{0, b}$ and $F_{0, c}$. The corresponding topologies are depicted in appendix C. For simplicity, the overall coupling factors, colour factors and factor of $(-1)$ due to fermion loop are removed from the tensor integrals. In particular, the quantities directly calculated via PYSECDEC are $\tilde{F}_{0, a}, \tilde{F}_{2, a}$ and $\tilde{F}_{0, b}$, which are related to $F_{0, a}, F_{2, a}, F_{0, b}$ and $F_{0, c}$ through following relations:

$$
\begin{aligned}
F_{0, a} & =\frac{1}{2} g_{s}^{2} \frac{m_{t}^{2}}{v^{2}}(6 \lambda v)^{2}\left(\frac{i}{16 \pi^{2}}\right)^{2} \frac{1}{2} \tilde{F}_{0, a}, \\
F_{2, a} & =\frac{1}{2} g_{s}^{2} \frac{m_{t}^{2}}{v^{2}}(6 \lambda v)^{2}\left(\frac{i}{16 \pi^{2}}\right)^{2} \frac{1}{2} \tilde{F}_{2, a}, \\
F_{0, b} & =\frac{1}{2} g_{s}^{2} \frac{m_{t}^{2}}{v^{2}}(6 \lambda)\left(\frac{i}{16 \pi^{2}}\right)^{2} \frac{1}{2} \tilde{F}_{0, b}, \\
F_{0, c} & =\frac{1}{2} g_{s}^{2} \frac{m_{t}^{2}}{v^{2}}(6 \lambda v)^{2} \frac{1}{s-m_{H}^{2}}\left(\frac{i}{16 \pi^{2}}\right)^{2} \frac{1}{2} \tilde{F}_{0, b} .
\end{aligned}
$$

In order to improve on the speed and convergence of the numerical evaluation, further measures are taken. First, only the finite parts are evaluated. To do this correctly, the integrands generated by PYSECDEC are multiplied with their prefactors, containing $\mathcal{O}(\epsilon)$ terms, before the integration. Nevertheless, we have cross-checked for specific phase-space points that UV-divergencies cancel for each diagram, although individual integral expressions can be separately UV-divergent.

Second, all integrals with the same denominator structure are added together before numerical integration. We have checked that the summation of several denominator structures prior to numerical integration does not lead to a faster convergence.

Third, different integrators were chosen for different integrals. A deterministic integrator like Cuhre [87], which is part of the CuBA library [88] and linked to PYSECDEC, is generally very fast and accurate for integrals with up to 5 dimensions. Beyond 5 dimensions, the integrator Vegas [89] is chosen. Furthermore, both Vegas and Cuhre give a $\chi^{2}$ estimate, stating the probability that the uncertainty associated to the result is accurate. Tests have repeatedly shown that the CuHRE results can be trusted only if $\chi^{2}$ is well below 1. Therefore, a routine was included to reperform the numerical integration with the more adaptive but generally slower integrator VEGAs when the $\chi^{2}$ value is too high. With this procedure we minimise cancellations and make sure that our numerical result is stable.

We have already mentioned that the UV finiteness of the form factors has been explicitly verified. Further tests have also been performed in order to ensure the correctness of the calculation. We have cross-checked the large $m_{t}$ limits for the $(b)$ and $(c)$ topologies (box-triangle) against analytical results. By setting $s_{12}=m_{H}^{2}$ we have found perfect agreement with the expression given in ref. [62]. Also, for the (a) topology (double-box), we have numerically tested that by artificially setting to $m_{X}$ the mass in the Higgs propagator connecting the two final-state Higgs, denoting the amplitude as $\mathcal{M}_{a, X}$, we obtain $\mathcal{M}_{a, X} \rightarrow \mathcal{M}_{b}\left[-2 \lambda_{3}^{2} /\left(\lambda_{4} m_{X}^{2}\right)\right]$ in the limit $m_{X} \rightarrow \infty$. In other words, by integrating out the heavy state $X$, the $(a)$ topology reduces to the $(b)$ topology where as expected the quar- 



Figure 4. The grid inputs for the real (left) and imaginary (right) parts of $\tilde{F}_{0, b}$.

tic coupling is an effective coupling $\lambda_{4}=-2 \lambda_{3}^{2} / m_{X}^{2}$. The factor of 2 originates from the number of diagrams contributing to the double-box amplitude, which is twice the number of diagrams contributing to the box-triangle amplitude.

\subsubsection{Grids for phase-space integration}

Up to this point we have discussed the strategy used for the evaluation of non-factorisable terms for a given phase space point. However, in this work we are interested in phenomenological predictions at colliders. Thus, the partonic squared matrix-elements have to be integrated over the phase-space and convoluted with parton-distribution-functions (PDFs). To this purpose, given the limited speed in the evaluation of the non-factorisable factors, it is helpful to build a grid that can be interpolated and quickly integrated over the relevant phase-space. In the following we explain how we have generated these grids, which have then been used together with an in-house Monte Carlo for obtaining the phenomenological results of section 4 .

Let us start by discussing the spin-0 component at two loops. The form factor $\tilde{F}_{0, b}$, corresponding to box-triangle diagrams, topologies $(b)$ and $(c)$, depends on only one kinematic variable $s$, hence a one-dimensional grid is sufficient. Thus, we have sampled several values of $s$ and performed a linear interpolation. As can be seen in figure $4, \tilde{F}_{0, b}$ features branch points at the thresholds $\sqrt{s}=2 m_{H}$ and $\sqrt{s}=2 m_{t}$.

On the contrary, the double box diagrams, topology (a), depend on both $s$ and the angle $\theta$ between $p_{1}$ and $p_{3}$. However, the dependence of $\tilde{F}_{0, a}$ on $\theta$ is actually small and it can be approximated by the first few terms in the partial wave expansion [90] of $\tilde{F}_{0, a}$ as

$$
\tilde{F}_{0, a}(s, \theta)=\sum_{i=0}^{\infty} a_{i}^{\prime}(s) d_{0,0}^{i}(\theta)=\sum_{i=0}^{\infty} a_{i}(s) P_{i}(\cos \theta) .
$$

We truncate the expansion in order to approximate the full results. We find that the $\theta$ dependence is weak, especially for $s_{12}<4 m_{H}^{2}$, i.e., below the top-pair threshold in the loops. In this phase-space region the top-quark loop can be integrated out, obtaining an effective $\mathrm{HHgg}$ coupling among the Higgs bosons and the gluons. With such an EFT description in the $m_{t} \rightarrow \infty$ limit there is no $\theta$ dependence. Thus, the dominant contribution originates from the term without $\theta$ dependence, namely, the $a_{0}(s)$ term. In order to have 

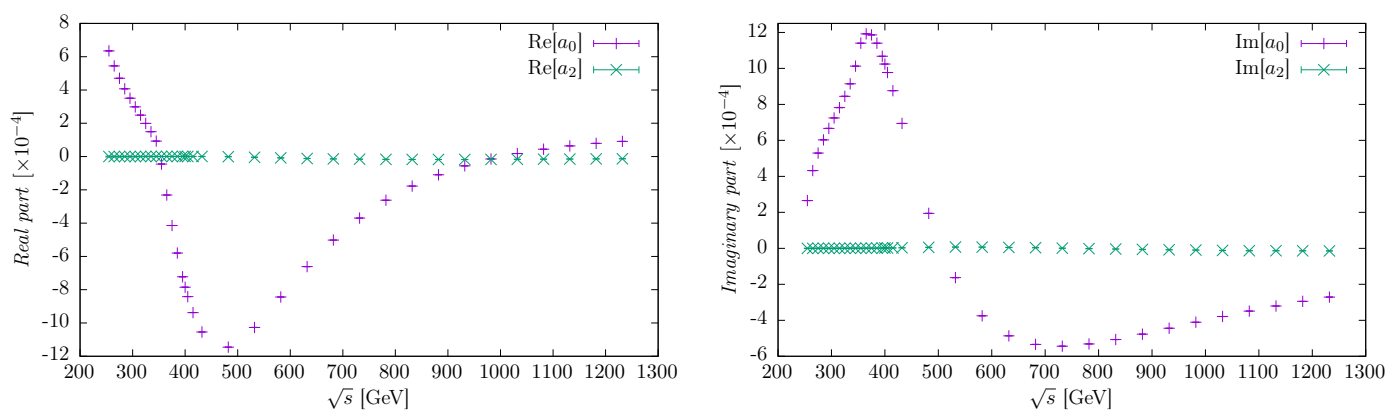

Figure 5. Fit results: real (left) and imaginary (right) parts of $a_{0}(s)$ and $a_{2}(s)$.

the $\theta$ dependence under control and to test the validity of the partial wave expansion, we do not only include the first term but also the second term, ${ }^{7}$

$$
\tilde{F}_{0, a}(s, \theta) \approx a_{0}(s)+a_{2}(s) P_{2}(\cos \theta)
$$

For each value of $s$, different values of $\theta$ have been sampled in order to perform a linear regression of $a_{0}(s)$ and $a_{2}(s)$. Afterwards, a linear interpolation is separately performed on both the values of $a_{0}(s)$ and $a_{2}(s)$. The validity of the truncation of the partial-wave expansion at $a_{2}(s)$ has also been investigated. First of all, we found that both the real and imaginary parts of $a_{2}(s)$ are substantially smaller than those of $a_{0}(s)$, as can be seen in figure 5 . Thus, contributions from higher-order $a_{i}(s)$ terms are expected to be even smaller than $a_{2}(s)$. Moreover we have estimated their contribution by comparing the value obtained with the approximation in eq. (2.38) after the regression and the actual value obtained. We can conclude that the truncation uncertainty is at the $\mathcal{O}(1 \%)$ level. Similarly to the case of $\tilde{F}_{0, b}$, also $a_{0}(s)$ (and $a_{2}(s)$ ) features branch points at the thresholds $\sqrt{s}=2 m_{H}$ and $\sqrt{s}=2 m_{t}$.

Let us conclude this section by commenting on the spin- 2 contribution $F_{2, a}$. Although there is a large dependence on $\theta$, we have verified that its contribution is strongly suppressed w.r.t. the spin- 0 contribution. For this reason we safely ignore this contribution in our phenomenological study of section 4 .

\section{Numerical results}

In this section we discuss the numerical results obtained for the $m(H H)$ distribution and the total rates at different collider energies. The phenomenological analyses of section 4 are based on these results.

In our calculation, we have used the following input parameters for the masses of the heavy SM particles,

$$
m_{t}=173.2 \mathrm{GeV}, \quad m_{W}=80.385 \mathrm{GeV}, \quad m_{Z}=91.1876 \mathrm{GeV}, \quad m_{H}=125.09 \mathrm{GeV},
$$

\footnotetext{
${ }^{7}$ Since $g g \rightarrow H H$ is by definition symmetric, the $a_{i}(s)$ coefficients are zero for odd values of $i$.
} 


\begin{tabular}{|c|c|c|c|}
\hline$\sqrt{s}[\mathrm{TeV}]$ & $\sigma_{0}[\mathrm{fb}]$ & $\sigma_{1}[\mathrm{fb}]$ & $\sigma_{2}[\mathrm{fb}]$ \\
\hline 14 & 19.49 & -15.59 & 5.414 \\
& - & $(-80.0 \%)$ & $(27.8 \%)$ \\
\hline 27 & 78.30 & -59.39 & 19.58 \\
& - & $(-75.8 \%)$ & $(25.0 \%)$ \\
\hline 100 & 790.8 & -556.8 & 170.8 \\
& - & $(-70.5 \%)$ & $(21.6 \%)$ \\
\hline
\end{tabular}

Table 1. LO contributions to $\sigma_{\mathrm{NLO}}^{\text {pheno }}$. We show for every entry the ratio with $\sigma_{0}$ at the same energy.

\begin{tabular}{|c|c|c|c|c|c|c|}
\hline$\sqrt{s}[\mathrm{TeV}]$ & $\tilde{\sigma}_{20}[\mathrm{fb}]$ & $\sigma_{30}[\mathrm{fb}]$ & $\sigma_{40}[\mathrm{fb}]$ & $\sigma_{01}[\mathrm{fb}]$ & $\sigma_{11}[\mathrm{fb}]$ & $\sigma_{21}[\mathrm{fb}]$ \\
\hline 14 & 0.7112 & -0.5427 & 0.0620 & 0.3514 & -0.0464 & -0.1433 \\
& $(3.6 \%)$ & $(-2.8 \%)$ & $(0.3 \%)$ & $(1.8 \%)$ & $(-0.2 \%)$ & $(-0.7 \%)$ \\
\hline 27 & 2.673 & -1.936 & 0.2102 & 1.3552 & -0.137 & -0.5127 \\
& $(3.4 \%)$ & $(-2.5 \%)$ & $(0.3 \%)$ & $(1.7 \%)$ & $(-0.2 \%)$ & $(-0.7 \%)$ \\
\hline 100 & 24.55 & -16.53 & 1.663 & 12.932 & -0.88 & -4.411 \\
& $(3.1 \%)$ & $(-2.1 \%)$ & $(0.2 \%)$ & $(1.6 \%)$ & $(-0.1 \%)$ & $(-0.6 \%)$ \\
\hline
\end{tabular}

Table 2. Two-loop contributions to $\sigma_{\mathrm{NLO}}^{\text {pheno }}$. We show for every entry the ratio with $\sigma_{0}$ at the same energy.

whereas all the other masses are set equal to zero. Similarly to ref. [68], we renormalise the fine-structure constant $\alpha$ in the $G_{\mu}$-scheme and we use as input parameter

$$
G_{\mu}=1.1663787 \cdot 10^{-5} \mathrm{GeV}^{-2} \text {. }
$$

The renormalisation scale for $\alpha_{s}$ and factorisation scale are set to be $\mu_{R}=\mu_{F}=\frac{1}{2} m(H H)=$ $\frac{1}{2} \sqrt{\hat{s}}$, and we have used the Parton-Distribution-Functions (PDF) set CT14LO [91]. We remind the reader that in our calculation we renormalise $\bar{c}_{6}$ in the $\overline{\mathrm{MS}}$ scheme and we set the renormalisation scale to $\mu_{\mathrm{EFT}}=2 m_{H}$. Moreover, we assume both the Wilson coefficients $\bar{c}_{6}$ and $\bar{c}_{8}$ at the scale $\mu_{\mathrm{EFT}}$.

In table 1 , we list the three different $\sigma_{i}$ contributions entering the LO part of $\sigma_{\mathrm{NLO}}^{\text {pheno }}$ at 14,27 and $100 \mathrm{TeV}$ proton-proton collisions. Similarly, in table 2 we list all the twoloop $\sigma_{i j}$ contributions entering $\sigma_{\mathrm{NLO}}^{\text {pheno }}$. We display in parentheses also their ratio with the $\mathrm{LO}$ prediction in the SM, $\sigma_{0}=\sigma_{\mathrm{LO}}^{\mathrm{SM}}$. As can be seen in tables 1 and 2 , cross sections considerably grow with the energy, while all the contributions induced by $\bar{c}_{6}$ and $\bar{c}_{8}$ mildly decrease in comparison with $\sigma_{\mathrm{LO}}^{\mathrm{SM}}$. Indeed, at large energies, the one-loop box diagrams is dominant w.r.t. the one with a triangle, which is the only one leading to $\bar{c}_{6}$ contributions at $\mathrm{LO}$ and to $\bar{c}_{8}$ contributions via loop corrections.

In figure 6 we show four different contour plots for the $14 \mathrm{TeV}$ energy. The upper plots show the ratio $\sigma_{\mathrm{NLO}}^{\mathrm{pheno}} / \sigma_{\mathrm{LO}}^{\mathrm{SM}}$, i.e., the ratio between our phenomenological prediction and the SM one, while the lower plots show the ratio $\sigma_{\mathrm{NLO}}^{\text {pheno }} / \sigma_{\mathrm{LO}}$, which corresponds to the $K$-factor from NLO corrections in our calculations. The left plots display these ratios in 

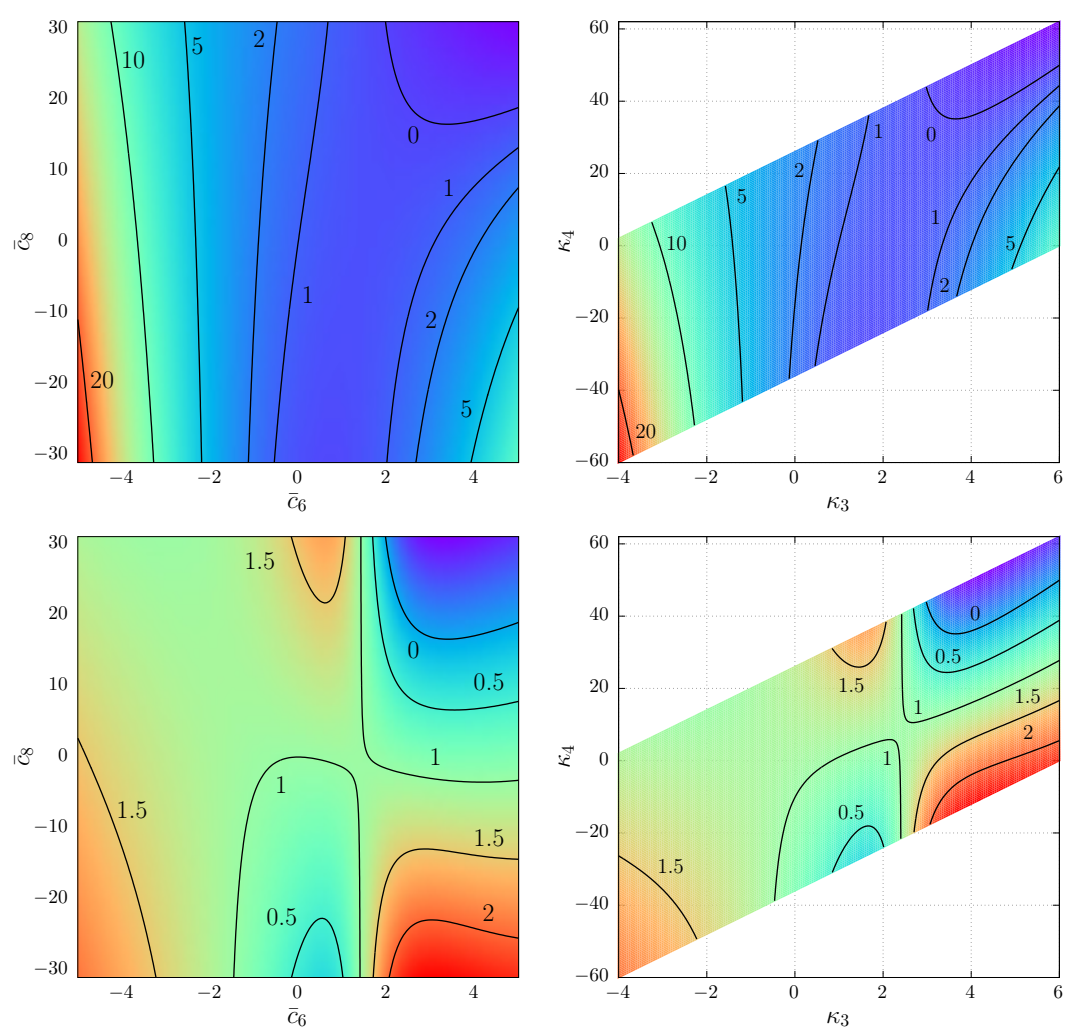

Figure 6. Contour plots at $14 \mathrm{TeV}$ for $\sigma_{\mathrm{NLO}}^{\text {pheno }} / \sigma_{\mathrm{LO}}^{\mathrm{SM}}(\mathrm{top})$ and $\sigma_{\mathrm{NLO}}^{\text {pheno }} / \sigma_{\mathrm{LO}}$ (bottom). Left plots show results in the $\left(\bar{c}_{6}, \bar{c}_{8}\right)$ plane, while right plots in the $\left(\kappa_{3}, \kappa_{4}\right)$ plane.

the $\left(\bar{c}_{6}, \bar{c}_{8}\right)$ plane, while the right plots in the $\left(\kappa_{3}, \kappa_{4}\right)$ one. In the plots we consider the perturbativity regime $\left|\bar{c}_{6}\right|<5$ and $\left|\bar{c}_{8}\right|<31$, which leads to values of $\left|\kappa_{4}\right|$ up to $\sim 60$. The upper plots show that large values of $\kappa_{3}$ can considerably enhance the value of the total cross section. For $\bar{c}_{6}<0$ there is only a small dependence on $\bar{c}_{8}$, while for $\bar{c}_{6}>0$ the dependence is sizable, and it even leads to negative cross sections for both large and positive $\bar{c}_{6}$ and $\bar{c}_{8}$. These effects are induced by the loop corrections; the LO predictions cannot be negative since they originate from a squared amplitude. It can be seen also in the lower plots where the contour line for $\sigma_{\mathrm{NLO}}^{\mathrm{pheno}} / \sigma_{\mathrm{LO}}=0$ is the same of $\sigma_{\mathrm{NLO}}^{\mathrm{pheno}} / \sigma_{\mathrm{LO}}^{\mathrm{SM}}=0$ in the upper plots. For negative values our prediction is unphysical, so it cannot be used for phenomenological studies. This is caused by the sum of $\bar{c}_{6}$ and $\bar{c}_{8}$ two-loop effects, which is large in absolute value. For the same reason also a region with $\sigma_{\mathrm{NLO}}^{\text {pheno }} / \sigma_{\mathrm{LO}}>2$ is present for large and positive(negative) $\bar{c}_{6}\left(\bar{c}_{8}\right)$. However, we do not exclude it since it is simply denoting a large NLO $K$-factor.

We move now to the differential distributions. In figure 7 we show the individual $\sigma_{i}$ (upper plot) and $\sigma_{i j}$ contributions (lower plots) to the $m(H H)$ distribution at $14 \mathrm{TeV} .{ }^{8} \mathrm{In}$ the case of negative values we plot their absolute values and display the result as a dashed line. Moreover, we show in figure 8 the ratio of any $\sigma_{i}$ and $\sigma_{i j}$ contribution over $\sigma_{\mathrm{LO}}^{\mathrm{SM}}$. In

\footnotetext{
${ }^{8}$ Besides an overall rescaling of the normalisation, distributions are very similar at $100 \mathrm{TeV}$ so we do not show them.
} 



Figure 7. Individual $\sigma_{i(j)}$ contributions at $14 \mathrm{TeV}$ as function of $m(H H)$. The upper plot display contributions to $\sigma_{\mathrm{LO}}$, while the lower plots those to $\Delta \sigma_{\bar{c}_{6}}$ (left) and to $\Delta \sigma_{\bar{c}_{8}}$ (right).

any plot this ratio is displayed as a black line, while we show in green the same result at the inclusive level, i.e., the values in parentheses in tables 1 and 2 . We observe that the $\bar{c}_{6}$ - and $\bar{c}_{8}$-induced contributions are most important close to threshold. Moreover, the quantities $\sigma_{1}, \sigma_{30}$ and $\sigma_{21}$ are negative. Therefore, large cancellations are present and shapes strongly depend on the value of $\bar{c}_{6}$ and loop corrections also on $\bar{c}_{8}$. In order to better show this point, in figure 9 we plot $\sigma_{\mathrm{LO}}$ for representative values of $\bar{c}_{6}$, namely, $\bar{c}_{6}= \pm 1, \pm 2, \pm 4$. Moreover, we plot the quantities $\Delta \sigma_{\bar{c}_{6}}$ and $\Delta \sigma_{\bar{c}_{8}} / \bar{c}_{8}$ from eqs. (2.12) and (2.13). As already explained, $\Delta \sigma_{\bar{c}_{6}}$ and $\Delta \sigma_{\bar{c}_{8}} / \bar{c}_{8}$ correspond to the loop corrections induced by $\bar{c}_{6}$ on top of $\bar{c}_{6}$ and the two-loop $\bar{c}_{8}$-dependent part, respectively. The normalisation and shape of $\sigma_{\mathrm{LO}}$ strongly depend on $\bar{c}_{6}$. The difference in shape is crucial in order to discriminate $\bar{c}_{6}$ values leading to the same total cross section and it is exploited in our work, which is based on the analysis of the $m(H H)$ distribution. The $\Delta \sigma_{\bar{c}_{6}}$ corrections grow for large $\left|\bar{c}_{6}\right|$ and the impact of $\Delta \sigma_{\bar{c}_{8}} / \bar{c}_{8}$ is larger for negative values of $\bar{c}_{6}$. In both cases, the largest effects are close to the threshold, as expected. 

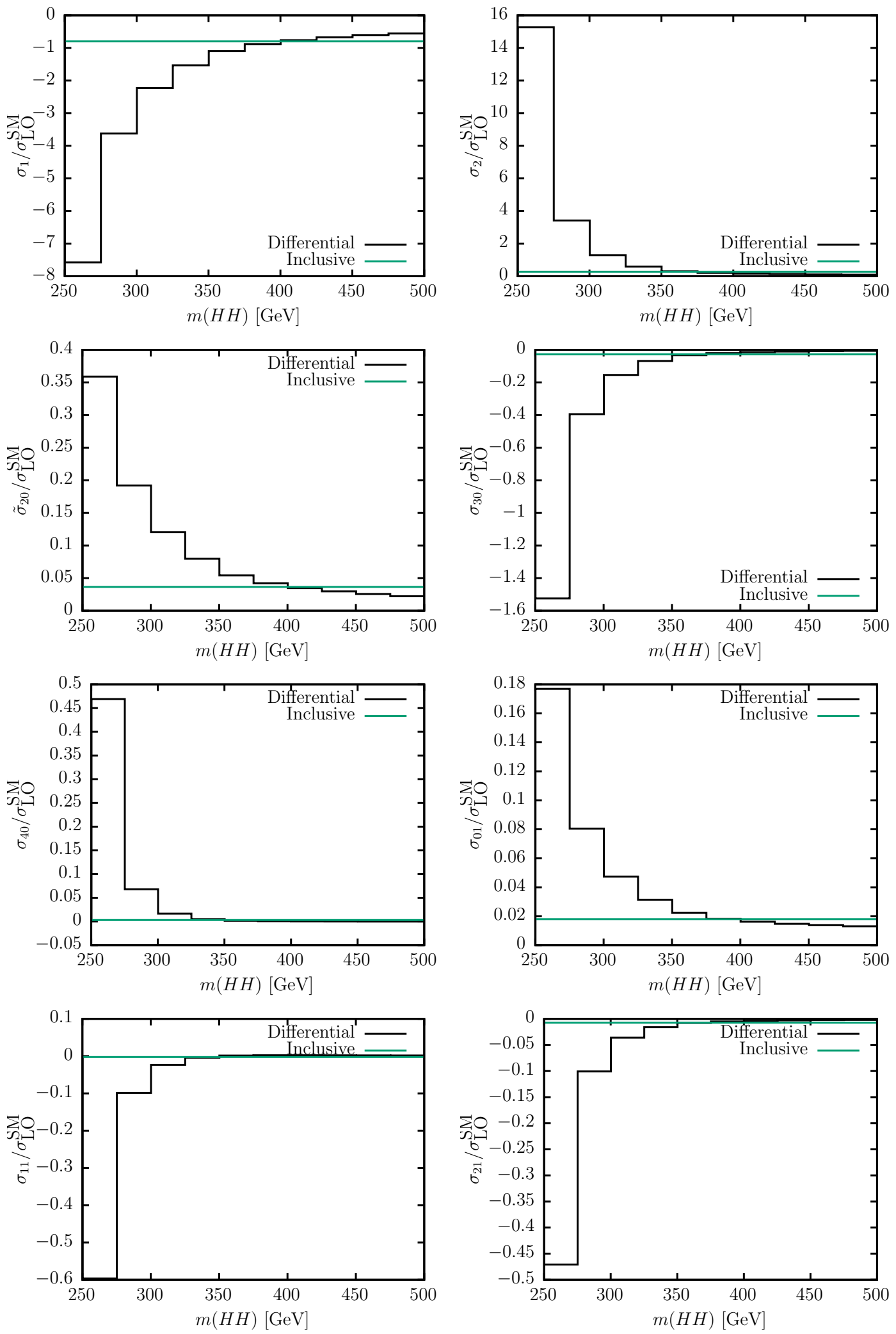

Figure 8. Relative impact of the $\sigma_{i(j)}$ contributions to the $m(H H)$ distribution at $14 \mathrm{TeV}$ (black) compared to the same quantity at total cross section level (green). 

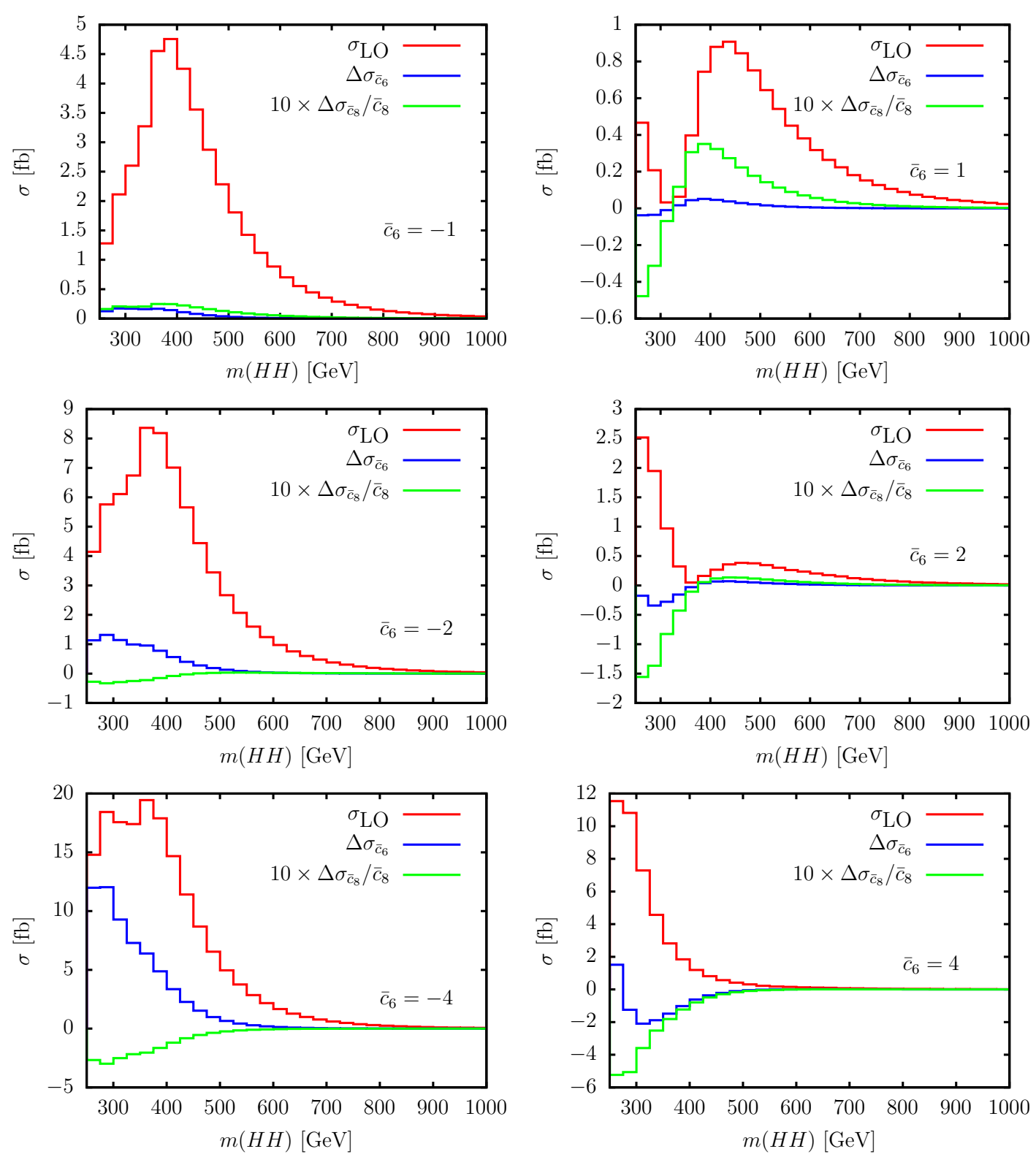

Figure 9. Different contributions $\left(\sigma_{\mathrm{LO}}, \Delta \sigma_{\bar{c}_{6}}\right.$ and $\left.\Delta \sigma_{\bar{c}_{8}} / \bar{c}_{8}\right)$ to the $m(H H)$ distribution at $14 \mathrm{TeV}$ for different $\bar{c}_{6}$ values.

\section{Constraints on the Higgs self couplings}

\subsection{General set up}

In this section we discuss the $\bar{c}_{6}$ and $\bar{c}_{8}\left(\kappa_{3}\right.$ and $\left.\kappa_{4}\right)$ constraints that can be derived from the measurements of double Higgs production in proton-proton collisions at the LHC and a $100 \mathrm{TeV}$ future collider. We consider the $b \bar{b} \gamma \gamma$ signature, which has been identified as the most promising channel and allow for the reconstruction of the di-Higgs invariance mass $m(H H)$. In order to be close to a realistic experimental analysis, we follow the study of 
ref. [21] for the case of HL-LHC and $100 \mathrm{TeV}$ collisions with $30 \mathrm{ab}^{-1}$ of luminosity. ${ }^{9} \mathrm{We}$ use the same selection cuts for the $b \bar{b} \gamma \gamma$ signature, we divide the reconstructed $m(H H)$ distribution in the same six bins and for each bin we take directly from ref. [21] the predictions for the background and for the signal in the SM. Results in ref. [21] take into account higher-order QCD corrections for both the signal and the background and also showering, hadronisation and detector effects. As already said, in our analyses we assume that $\bar{c}_{6}$ and $\bar{c}_{8}$ effects factorise QCD corrections and we compute the effects of selection cuts (see appendix A) adding $H$ decays at the parton level. Thus, we also assume that showering, hadronisation and detector effects factorise the effect of selections cuts on the $b \bar{b} \gamma \gamma$ signature. Moreover, although there are in principle also $\bar{c}_{6}$ effects from $H \rightarrow b \bar{b}$ and $H \rightarrow \gamma \gamma$ decays, they are actually completely negligible. Indeed, as can be seen in ref. [62], for $\left|\bar{c}_{6}\right|<5$ the predictions for the corresponding branching ratios deviate at most by $1 \%$. Especially, they are very close in absolute value and with opposite sign. Thus, the net effect is at most at the permille level.

In order to set limits on $\bar{c}_{6}$ and $\bar{c}_{8}$ we perform a $\chi^{2}$ fit on the $m(H H)$ distribution. For simplicity, as done in ref. [21], we will include statistical uncertainties only. The impact of theoretical uncertainties and experimental systematic uncertainties is expected to be much smaller than statistical ones [21, 72], therefore they would not in general lead to significant differences; some caveats are present for the $100 \mathrm{TeV}$ case and will be discussed afterwards. On the other hand, we have found that assuming $\bar{c}_{6}$ and $\bar{c}_{8}$ effects as flat within each of the six bins of the reconstructed $m(H H)$ distributions can strongly distort the results. Indeed, in each $m(H H)$ bin, $\bar{c}_{6}$ and $\bar{c}_{8}$ effects are not flat over the full $b \bar{b} \gamma \gamma$ phase-space. Thus, selection cuts have an impact not only on the total number of events observed but also on the ratio $\sigma_{\mathrm{NLO}}^{\mathrm{pheno}} / \sigma_{\mathrm{LO}}^{\mathrm{SM}}$. More details about the fit procedure can be found in appendix B.

Similarly to what has been in done in ref. [68], we consider two different scenarios for setting bounds on Higgs self couplings:

\section{Scenario 1: Well-behaved EFT $\left(\kappa_{3} \neq 1, \kappa_{4} \sim 6 \kappa_{3}-1\right)$.}

The contribution from $\bar{c}_{8}$ is suppressed w.r.t. the one from $\bar{c}_{6}$, hence we can safely set $\bar{c}_{8}=0$. We do not assume only $\bar{c}_{6} \sim 0$, i.e., an SM-like configuration, but also allow for large BSM effects $\left(\left|\bar{c}_{6}\right| \gtrsim 0\right)$.

\section{Scenario 2: General parameterisation allowing for $\kappa_{3} \neq 1$ and $\kappa_{4} \neq 6 \kappa_{3}-1$.}

Effects from $\bar{c}_{8}$ are not negligible and therefore we consider $\bar{c}_{8} \neq 0$. Also in this case, we consider $\bar{c}_{6} \sim 0$ or $\left|\bar{c}_{6}\right| \gtrsim 0$, allowing for large BSM effects.

In Scenario 1, assuming that Nature corresponds to $\bar{c}_{6}=\bar{c}_{6}^{\text {true }}$, we will analyse the constraints that can be set on $\bar{c}_{6}$. In the Scenario 2 , setting $\bar{c}_{8}^{\text {true }}=0$, we explore the constraints that can be set on the $\left(\bar{c}_{6}, \bar{c}_{8}\right)$ plane, for different value of $\bar{c}_{6}^{\text {true }}$. One may be tempted to study also a "Scenario 3", as done in ref. [72], where $\bar{c}_{6}=0$ and $\bar{c}_{8} \neq 0$.

\footnotetext{
${ }^{9}$ In principle, also the analysis in ref. [36] can be used. However, the amount of details provided by the authors is not sufficient for performing our study. For the same reason, we do not show results at $27 \mathrm{TeV}$ in our paper, although may be extracted performing the analysis in ref. [36].
} 

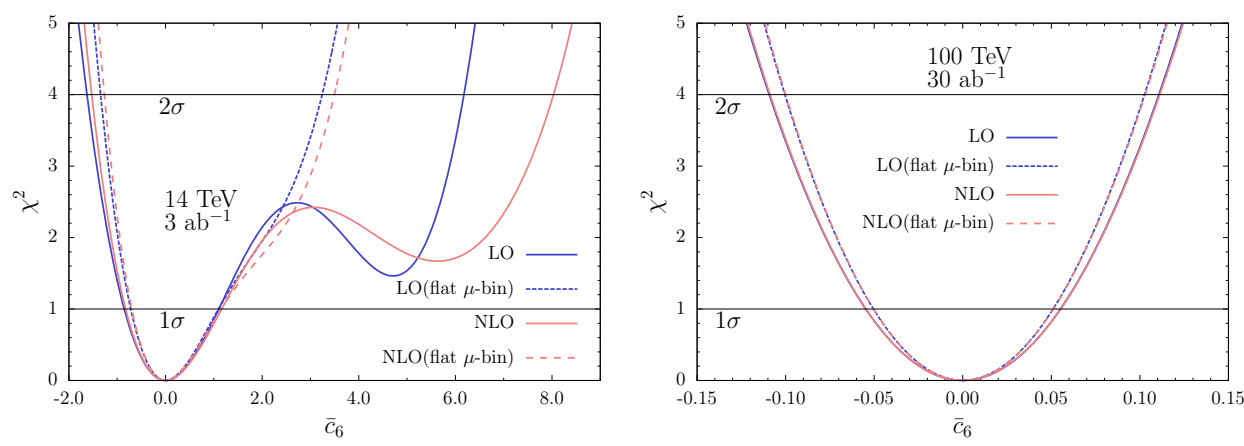

Figure 10. $\chi^{2}$ as a function of $\bar{c}_{6}$ for $\bar{c}_{8}=0$ at 14 (left) and 100 (right) TeV.

However, this configuration is unstable. Indeed, it is easily spoiled by the running of $\bar{c}_{6}$ and $\bar{c}_{8}$ at different scales, ${ }^{10}$ since it is not protected by any symmetry and not emerging from an EFT expansion. For this reason we refrain from considering this scenario.

\subsection{Scenario 1}

We start considering the $\chi^{2}$ function and the $1 \sigma$ and $2 \sigma$ bounds that can be obtained for $\bar{c}_{6}$ assuming $\bar{c}_{6}^{\text {true }}=0$, at the HL-LHC and at a future $100 \mathrm{TeV}$ collider. In figure 10 we plot the $\chi^{2}$ function ${ }^{11}$ using $\sigma_{\mathrm{NLO}}^{\text {pheno }}$ or $\sigma_{\mathrm{LO}}$ in the fit. Moreover, we show the relevance of fully differential information in the treatment of $\bar{c}_{6}$ and $\bar{c}_{8}$ effects. In the case denoted as "flat $\mu$-bin" in the plot, we assume that for each $m(H H)$-bin the impact of $\bar{c}_{6}$ effects can be evaluated via the ratio $\sigma / \sigma_{\mathrm{LO}}$ without taking into account the selection cuts on the $b \bar{b} \gamma \gamma$ final state, where $\sigma$ can be either $\sigma_{\mathrm{NLO}}^{\text {pheno }}$ or $\sigma_{\mathrm{LO}}$. We remark that both in the "flat $\mu$-bin" and normal cases, selection cuts are taken into account for the SM signal; the "flat $\mu$-bin" concerns only the modelling of $\bar{c}_{6}$ and $\bar{c}_{8}$ effects for the $m(H H)$-binning of the fit. More details are given in appendix B. As can be seen in figure 10, NLO effects, which in Scenario 1 corresponds to $\Delta \sigma_{\bar{c}_{6}}$ only, are relevant only for large values of $\bar{c}_{6}$. On the contrary, the "flat $\mu$-bin" assumption strongly distorts the $\chi^{2}$ profile, especially for positive values of $\bar{c}_{6}$. Indeed, as can be seen from the dashed lines, with this assumption the $2 \sigma$ bounds at $14 \mathrm{TeV}$ would be artificially improved. This effect is due to the fact that for $\bar{c}_{6} \gtrsim 2$ the bulk of events is in the first bin(s) of the $m(H H)$ distribution (see figure 9), but on the other hand selection cuts, which strongly depend on $m(H H)$, dramatically suppress events close to the threshold configuration (see appendix A).

Consistently taking into account the selection cuts in our analysis, we find the following $2 \sigma$ intervals,

$$
\begin{aligned}
-0.5<\kappa_{3} & =1+\bar{c}_{6}<8 & & \text { at } 14 \mathrm{TeV} \text { with } 3 \mathrm{ab}^{-1}, \\
0.9<\kappa_{3} & =1+\bar{c}_{6}<1.1 & & \text { at } 100 \mathrm{TeV} \text { with } 30 \mathrm{ab}^{-1} .
\end{aligned}
$$

Results at $14 \mathrm{TeV}$ in (4.1) are consistent with the ATLAS projections for the HL-LHC [92], which are also based on the $b \bar{b} \gamma \gamma$ signature and on statistical uncertainties only.

\footnotetext{
${ }^{10}$ As can be easily derived by the counterterm for $\bar{c}_{6}$ given in ref. [68], the one-loop $\beta$-function for $\bar{c}_{6}$ contains terms proportional to $\bar{c}_{8}$ and independent on $\bar{c}_{6}$.

${ }^{11}$ In fact, the plots display the quantity $\chi^{2}-\min \left(\chi^{2}\right)$. For brevity we will refer to it as $\chi^{2}$.
} 



Figure 11. Sensitivity ( $2 \sigma$ bounds) on $\bar{c}_{6}$ as function of $c_{6}^{\text {true }}$ for $14 \mathrm{TeV}$ (left) and $100 \mathrm{TeV}$ (right).

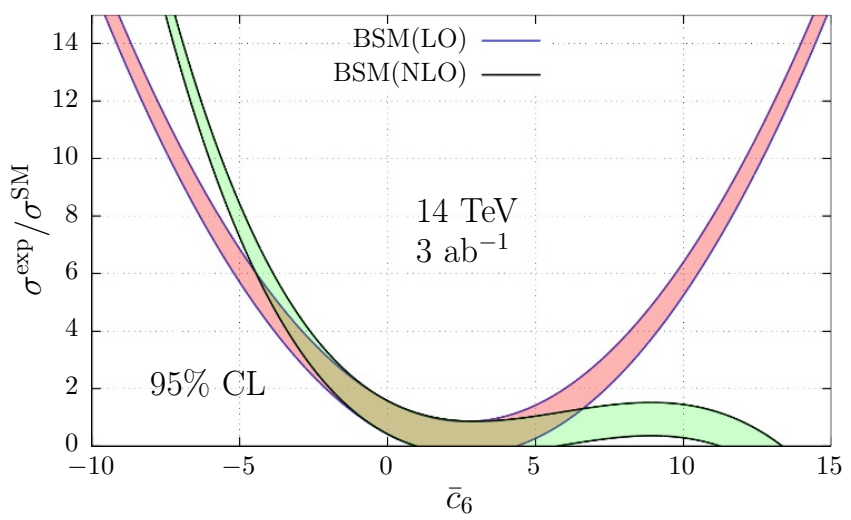

Figure 12. Bounds on $\bar{c}_{6}$ that can be set according to the supposedly double-Higgs measured cross section, normalised to the corresponding SM prediction. The red band is obtained considering only $\sigma_{\mathrm{LO}}$, while the green using $\sigma_{\mathrm{NLO}}^{\mathrm{pheno}}$.

We now move to the case where $\bar{c}_{6}^{\text {true }}$ can be different from zero. In figure 11 we show $2 \sigma$ bounds for $\bar{c}_{6}$ as a function of $\bar{c}_{6}^{\text {true }}$. It turns out that if $\bar{c}_{6}$ is negative, bounds can be sizeably stronger. For instance, assuming $\bar{c}_{6}^{\text {true }}=-2$ a limit $-1.5 .<\kappa_{3}=1+\bar{c}_{6}<-0.5$ can be obtained at HL-LHC, which is remarkably more stringent than in the $\bar{c}_{6}^{\text {true }}=0$ case of (4.1). In the case of $100 \mathrm{TeV}$, large and negative values of $\bar{c}_{6}^{\text {true }}$ seem to lead to subpercent precision. This should be interpreted as indication that high precision may be reached in this scenario, but also that theory and systematic uncertainties have to be taken into account to estimate a realistic value. In both the plots of figure 11 we show also results under the "flat $\mu$-bin" assumption as dashed lines. As can be seen, this assumption would have a strong effect to the $\bar{c}_{6}$ bounds, especially for $\bar{c}_{6} \gtrsim 0$.

In this context we want also to stress an important point that has been somehow overlooked in both theory and experimental studies on $\kappa_{3}$-determination. In figure 12 we plot the $2 \sigma$ constraints that can be obtained on $\bar{c}_{6}$ by varying of $\sigma^{\exp } / \sigma^{\mathrm{SM}}$, where $\sigma^{\exp }$ is the measured value and $\sigma^{\mathrm{SM}}$ is the SM prediction. We derive the constraints using two different approximations: $\sigma_{\mathrm{NLO}}^{\text {pheno }}$ and $\sigma_{\mathrm{LO}}$. As can be seen, for $\left|\bar{c}_{6}\right| \gtrsim 5$, where perturbativity is violated, the constraints on $\bar{c}_{6}$ strongly depend on the choice between $\sigma_{\mathrm{NLO}}^{\text {pheno }}$ and $\sigma_{\mathrm{LO}}$. When data are fitted with $\sigma_{\mathrm{LO}}$ predictions, $\bar{c}_{6}$ or equivalently $\kappa_{3}$ is a parameter of ignorance that only for $\left|\kappa_{3}-1\right|=\left|\bar{c}_{6}\right| \lesssim 5$ coincides to the quantity one is interested in. Outside this 

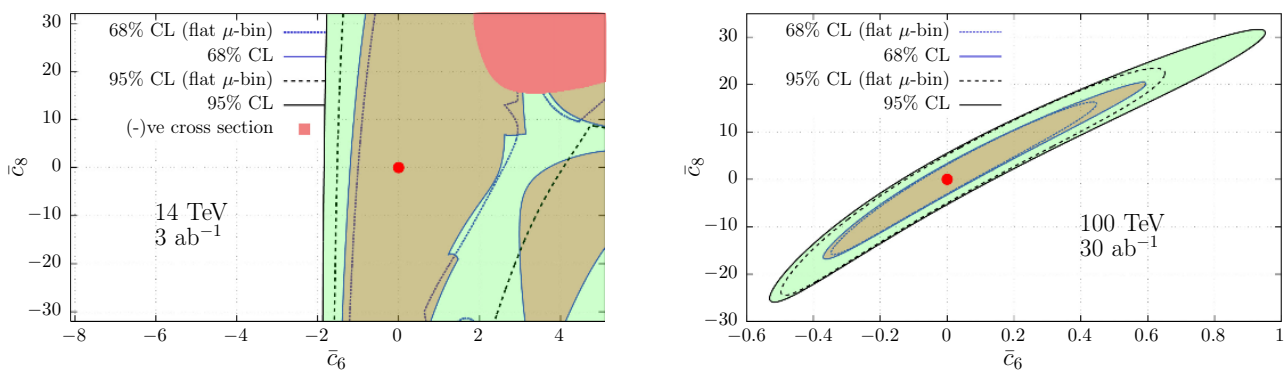

Figure 13. Expected $1 \sigma$ and $2 \sigma$ bounds in the $\left(\bar{c}_{6}, \bar{c}_{8}\right)$ plane at 14 (left) and $100 \mathrm{TeV}$ (right), assuming $\bar{c}_{6}^{\text {true }}=\bar{c}_{8}^{\text {true }}=0$ (denoted by red dots).

range, $\bar{c}_{6}\left(\right.$ or $\left.\kappa_{3}\right)$ is only suggesting how far from the SM predictions is the experimental result. The usage of $\sigma_{\mathrm{NLO}}^{\text {pheno }}$ or any higher-order corrections in the place of $\sigma_{\mathrm{LO}}$ is not improving this situation, since the regime is not perturbative for $\left|\bar{c}_{6}\right| \gtrsim 5$. In conclusion, one can set bounds outside the $\left|\kappa_{3}-1\right|=\left|\bar{c}_{6}\right| \lesssim 5$ range, but only within this region they properly refer to the quantities we are interested in and defined via parameters in the Lagrangian.

\subsection{Scenario 2}

This scenario allows us to discuss the most important phenomenological results of this work, i.e., the expected constraints on $\bar{c}_{6}$ and $\bar{c}_{8}\left(\kappa_{3}\right.$ and $\left.\kappa_{4}\right)$ that can be obtained via double Higgs production at HL-LHC and a $100 \mathrm{TeV}$ future collider. Assuming $\bar{c}_{8}^{\text {true }}=0$, these constraints are shown in the left and right plot of figure 13, respectively. We show $2 \sigma$ results and again the effect due to the "flat $\mu$-bin" assumption, the red area corresponds to the region where the cross-section is negative (cf. left plots in figure 6). As already mentioned, no phenomenological study can be performed in this configuration. Similarly, for a given $\left(\bar{c}_{6}, \bar{c}_{8}\right)$, predictions for some bins can be negative, while positive for others; we retain the information only for those bins where the cross-section is predicted to be positive. As can be seen from figure 13, at HL-LHC the presence of $\bar{c}_{8}$ contributions is not sizeably affecting the result in (4.1), obtained under the assumption $\bar{c}_{8}=0$. On the other hand, no sensible constraints can be obtained at the HL-LHC on the $\bar{c}_{8}$ parameter.

Results at $100 \mathrm{TeV}$ collisions are qualitatively very different than at the HL-LHC. The bounds on $\bar{c}_{6}$ are affected by the presence on $\bar{c}_{8}$. As can be seen from the right plot of figure 13 , the bounds are $0.4<\kappa_{3}=1+\bar{c}_{6}<2$, which is less precise than (4.2), obtained under the assumption $\bar{c}_{8}=0$. Although most of the perturbativity $\bar{c}_{8}$ region is not excluded, there is a clear direction in the contours of the constraints in the $\left(\bar{c}_{6}, \bar{c}_{8}\right)$ plane. We have also verified that assuming that the value of the Yukawa coupling of the top-quark is known with $1 \%$ precision [93] and is compatible with its SM prediction, if we take into account this uncertainty there are no visible difference in the right plot of figure $13 .^{12}$

In figures 14 and 15 we show the constraints that can be set in the $\left(\bar{c}_{6}, \bar{c}_{8}\right)$ plane assuming $\bar{c}_{8}^{\text {true }}=0$ and $\bar{c}_{6}^{\text {true }}= \pm 1, \pm 2, \pm 4$ for HL-LHC and a $100 \mathrm{TeV}$ collider, respectively.

\footnotetext{
${ }^{12}$ In order to perform this check, we have taken into account the effects of an anomalous Yukawa coupling at $\sigma_{\mathrm{LO}}$ level.
} 

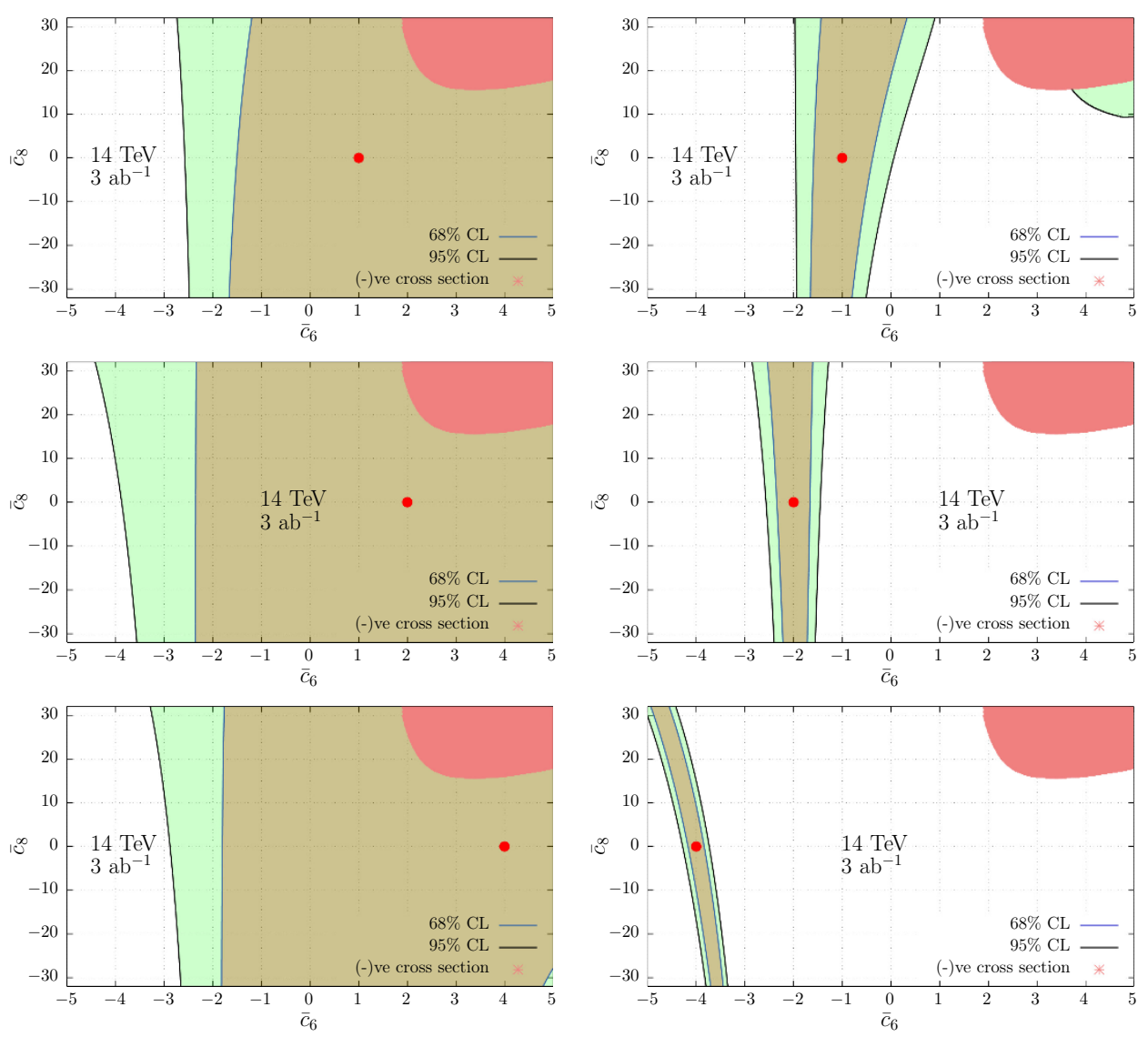

Figure 14. Expected $1 \sigma$ and $2 \sigma$ bounds in the $\left(\bar{c}_{6}, \bar{c}_{8}\right)$ plane at $14 \mathrm{TeV}$, assuming $\bar{c}_{6}^{\text {true }}= \pm 1, \pm 2, \pm 4$ and $\bar{c}_{8}^{\text {true }}=0$ (denoted by red dots).

As can be seen in figure 14, at HL-LHC for large and positive values of $\bar{c}_{6}^{\text {true }}$ we find results very close to $\bar{c}_{6}^{\text {true }}=0$. In general, including the case of negative $\bar{c}_{6}^{\text {true }}$ values, we see that limits on $\bar{c}_{6}$ are not sizeably affected by the presence of $\bar{c}_{8}$. However, sensible constraints on $\bar{c}_{8}$ cannot be obtained at the HL-LHC. At $100 \mathrm{TeV}$, figure 15 , (large) negative values of $\bar{c}_{6}^{\text {true }}$ lead to strong constraints in the $\left(\bar{c}_{6}, \bar{c}_{8}\right)$ plane. However, we remind the reader that we do not take into account theory and experimental systematic uncertainties. As said for the corresponding results in Scenario 1, these results may be affected by the aforementioned uncertainties. The case of $\bar{c}_{6}^{\text {true }}=4$, and in a smaller extent also the case $\bar{c}_{6}^{\text {true }}=2$, display $1 \sigma$ and $2 \sigma$ regions that are disconnected by the $\left(\bar{c}_{6}^{\text {true }}, \bar{c}_{8}^{\text {true }}\right)$ point. Those regions correspond to configurations where the first $m(H H)$ bin, but not all the others, is predicted to be negative by $\sigma_{\mathrm{NLO}}^{\text {pheno }}$. As explained, we remove its contribution to the $\chi^{2}$, which consequently is strongly reduced and therefore those regions cannot be excluded. The negative value of the first bin is also the origin of the sharp wiggling of the lines in the left plot of figure 13. In both cases we could have eliminated these effects by artificially setting $\sigma_{\mathrm{NLO}}^{\text {pheno }}$ to zero for negative values, but we preferred to be conservative and exclude the contribution to $\chi^{2}$ in these cases. 

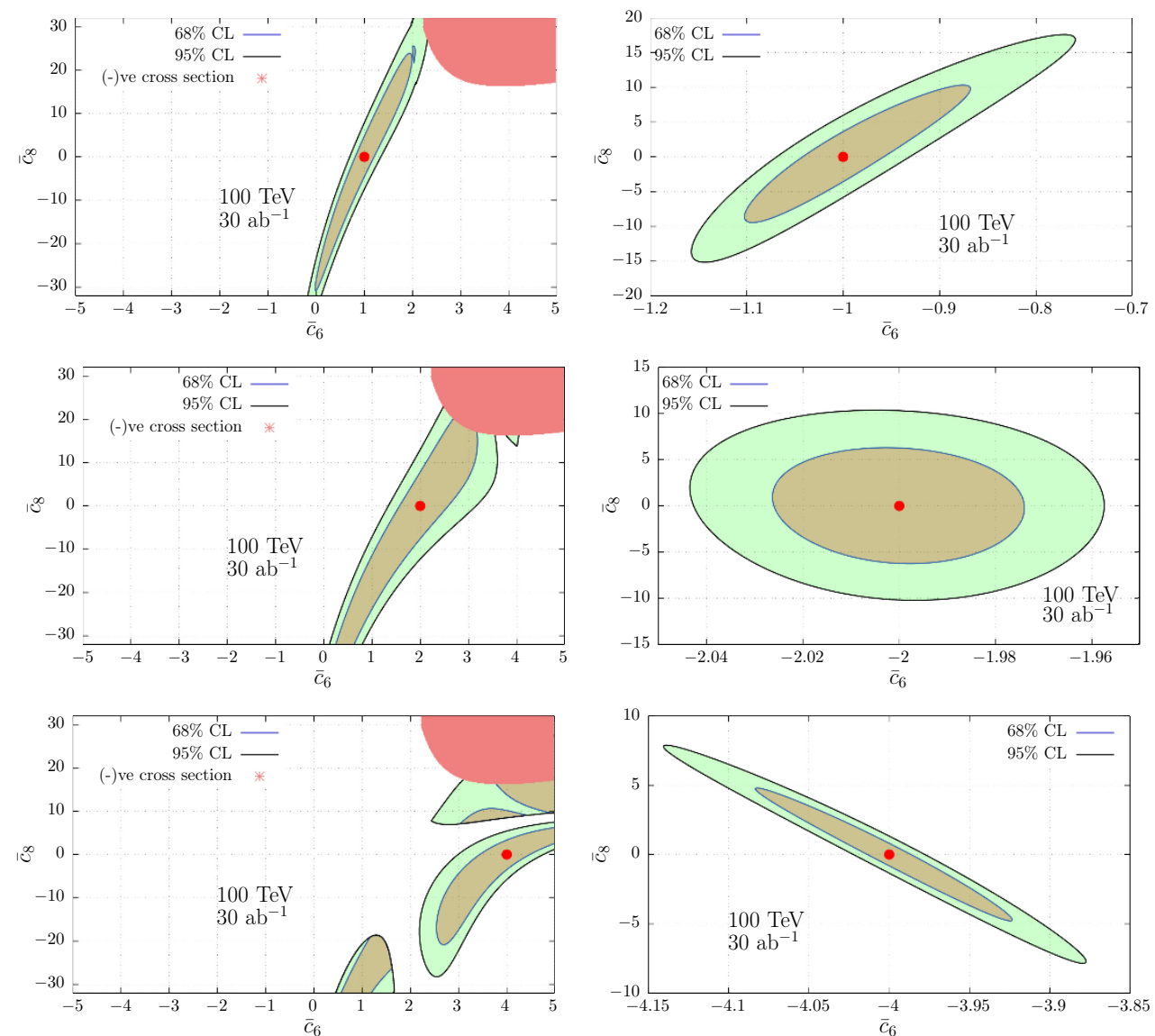

Figure 15. Expected $1 \sigma$ and $2 \sigma$ bounds in the $\left(\bar{c}_{6}, \bar{c}_{8}\right)$ plane at $100 \mathrm{TeV}$, assuming $\bar{c}_{6}^{\text {true }}= \pm 1, \pm 2$, \pm 4 and $\bar{c}_{8}^{\text {true }}=0$ (denoted by red dots).


Figure 16. Comparison between expected $2 \sigma$-bounds from $H H(b \bar{b} \gamma \gamma)$ and $H H H(b \bar{b} b \bar{b} \gamma \gamma)$ at $100 \mathrm{TeV}$. The right plot in $\left(\kappa_{3}, \kappa_{4}\right)$ plane takes in account the perturbativity bounds on $\bar{c}_{6}$ and $\bar{c}_{8}$.

Last but not least, in figure 16 we compare the constraints obtained for $\bar{c}_{6}^{\text {true }}=0$ at $100 \mathrm{TeV}$ (right plot of figure 13) with the corresponding ones obtained following the analysis in refs. [56, 94], based on the $b \bar{b} b \bar{b} \gamma \gamma$ signature emerging from $p p \rightarrow H H H$ production. ${ }^{13}$ Triple Higgs bounds are derived via two different assumptions on $b$-tagging efficiency: op-

\footnotetext{
${ }^{13}$ We have also looked at results from ref. [53]; following this analysis bounds are a bit stronger than in the case with $60 \%$ b-tagging efficiency.
} 
timistic (80\%) and conservative (60\%). As can be seen in figure 16, double Higgs bounds are stronger than those from triple Higgs with the optimistic assumption. Especially, they are complementary to those from triple Higgs with the conservative assumption and their combination can lead to stronger results. We also show the corresponding comparison in $\left(\kappa_{3}, \kappa_{4}\right)$ plane taking into account the perturbative bounds on $\bar{c}_{6}$ and $\bar{c}_{8}$.

\section{Conclusion}

The experimental determination of the Higgs potential and in particular of the Higgs self couplings is one the most far fetching goals of the HL-LHC and of future colliders. Its importance is matched only by the difficulty of such an endeavour: rates for multiple Higgs production which are directly sensitive to the self couplings, are very low making it hard to study distributions where most of the sensitivity actually lies. This is certainly true for the cubic coupling at the LHC, which can be accessed directly via $H H$ production, but becomes dramatic for the quartic coupling: its direct determination calls for measurements in the $\mathrm{HHH}$ final state, whose production rate will be small even at a future $100 \mathrm{TeV}$ proton-proton colliders.

The challenge on the one hand and the high-stakes on the other hand have provided strong motivation to the theoretical and experimental high-energy-physics community to devise alternative strategies. Among them, a new approach has emerged building up from the simple idea that single Higgs cross sections might display a sensitivity on the cubic coupling at higher orders. Since the first proposal in the context of future $e^{+} e^{-}$colliders [60], the idea has been developed and extended to hadron colliders, eventually proving to be competitive with the direct determinations. A very first experimental analysis by CMS [95] based on the proposal of refs. [62, 64] has confirmed the expectations of the theoretical studies.

Recently, some of us have proposed to further extend the idea and determine the (cubic and) quartic coupling exploiting the sensitivity coming from loop effects in $H H$ in the context of future $e^{+} e^{-}$colliders [68]. In this work we have moved one step further and explored the reach of hadron colliders by determining the sensitivity to the (cubic and) quartic coupling of the main double Higgs production channel, $g g \rightarrow H H$, up to two loops. Being a technically challenging two-loop computation we have employed the most up-to-date numerical multi-loop techniques, providing for the first time a complete and consistent calculation of these effects.

We have considered two different scenarios, one "EFT-like" where the cubic and quartic couplings are related and one where they are varied independently. Our results clearly indicate that while the HL-LHC will have limited sensitivity, at the FCC-100 the precision on $H H$ differential measurements could be such that $H H$ will be more sensitive to independent deviations in the self couplings than $\mathrm{HHH}$ production itself. The best constraints on the quartic will therefore be obtained by combining $H H$ precision measurements with the direct determinations from $\mathrm{HHH}$. 


\section{Acknowledgments}

We would like to thank Matthias Kerner for a valuable comment on the input files for the numerical integration of the two-loop amplitudes. S.B. would like to thank the hospitality of CP3 Louvain during the completion of the work. D.P. is supported by the Alexander von Humboldt Foundation, in the framework of the Sofja Kovalevskaja Award Project "Event Simulation for the Large Hadron Collider at High Precision". A.S. is supported by the MOVE-IN Louvain Cofund grant. The work of X.Z. is supported the European Union's Horizon 2020 research and innovation programme as part of the Marie Skłodowska-Curie Innovative Training Network MCnetITN3 (grant agreement no. 722104). This work has received funding from the ERC grant "MathAm" and from F.R.S.-FNRS under the 'Excellence of Science' EOS be.h project n. 30820817. Computational resources have been provided by the supercomputing facilities of the Université catholique de Louvain (CISM/UCL) and the Consortium des Équipements de Calcul Intensif en Fédération Wallonie Bruxelles (CÉCI).

\section{A Cut efficiency}

In this section we explicitly write the cuts used in our analysis. The cuts are the same of ref. [21], on which our analysis is based. Specifically, at $14 \mathrm{TeV}$, they correspond to

$$
\begin{aligned}
p_{T}\left(b_{1}\right) & >50 \mathrm{GeV}, & p_{T}\left(b_{2}\right) & >30 \mathrm{GeV}, \\
p_{T}\left(\gamma_{1}\right) & >50 \mathrm{GeV}, & p_{T}\left(\gamma_{2}\right) & >30 \mathrm{GeV}, \\
|\eta(b)| & <2.5, & & |\eta(\gamma)|<2.5, \\
0.5<\Delta R(b, b) & <2, & \Delta R(\gamma, \gamma) & <2, \\
\Delta R(b, \gamma) & >1.5, & &
\end{aligned}
$$

while at $100 \mathrm{TeV}$, the $p_{T}$ cuts are replaced by:

$$
\begin{array}{ll}
p_{T}\left(b_{1}\right)>60 \mathrm{GeV}, & p_{T}\left(b_{2}\right)>40 \mathrm{GeV}, \\
p_{T}\left(\gamma_{1}\right)>60 \mathrm{GeV}, & p_{T}\left(\gamma_{2}\right)>40 \mathrm{GeV} .
\end{array}
$$

In figure 17, we show the differential cut efficiency for the signal, assuming SM double Higgs production and narrow-width approximation. In other words, we plot the ratio between the number of events predicted in the SM with and without the cuts as function of $m(H H)$. Since spin-0 contributions dominate for both SM and BSM cases, cut efficiencies for BSM cases are very similar.

The zero efficiency in the $250 \mathrm{GeV}<m(H H)<300 \mathrm{GeV}$ phase-space region is not a surprise; when Higgs boson pairs are produced at the threshold, both the $b \bar{b}$ and $\gamma \gamma$ pairs from the Higgs decays are back-to-back and therefore rejected by the cuts $\Delta R(b, b)<2$ and $\Delta R(\gamma, \gamma)<2$. Increasing the energy, both Higgs can have non-vanishing transverse momentum and therefore their decay products can be not back-to-back and tend to be collimated for very high energies. 

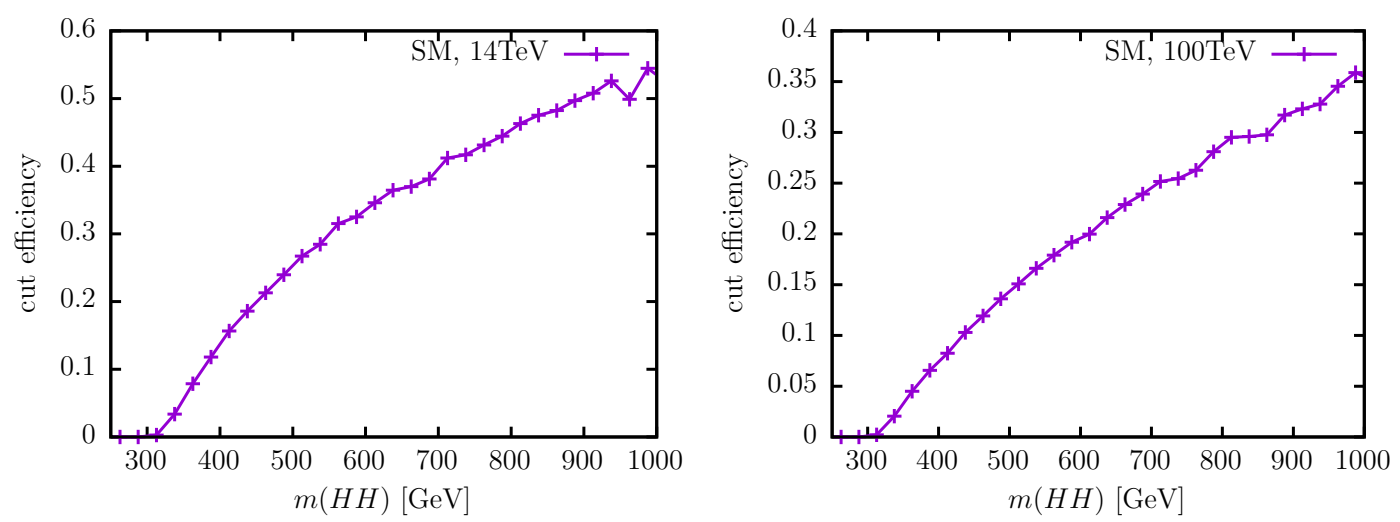

Figure 17. Differential cut efficiency for SM double-Higgs signal from the $b \bar{b} \gamma \gamma$ signature, at parton level.

\section{B Fit details}

In this appendix we describe in detail the $\chi^{2}$ functions that have been used in this work for extracting from $m(H H)$ distributions $1 \sigma$ and $2 \sigma$ bounds in the $\left(\bar{c}_{6}, \bar{c}_{8}\right)$ parameter space. The general formula of the $\chi^{2}$ that has been exploited for our results has two degrees of freedom, $\bar{c}_{6}$ and $\bar{c}_{8}$, and reads

$$
\chi^{2}=\sum_{i=1}^{n_{\text {bins }}} \frac{\left[N_{i}^{H H}\left(\bar{c}_{6}, \bar{c}_{8}\right)-N_{i}^{H H}\left(\bar{c}_{6}^{\text {true }}, \bar{c}_{8}^{\text {true }}\right)\right]^{2}}{N_{i}^{H H}\left(\bar{c}_{6}, \bar{c}_{8}\right)+N_{i}^{\text {BKG }}} \theta\left(N_{i}^{H H}\left(\bar{c}_{6}, \bar{c}_{8}\right)\right)
$$

where $\bar{c}_{8}^{\text {true }}$, and in some cases also $\bar{c}_{8}$, have been set equal to zero. On the contrary $\bar{c}_{6}^{\text {true }}$ has been fixed to different values in the $-5<\bar{c}_{6}<5$ range and $\bar{c}_{6}$ has been kept always free. In eq. (B.1), $N_{i}^{H H}\left(\bar{c}_{6}, \bar{c}_{8}\right)$ is the number of signal events in each bin $i$ for the specific $\bar{c}_{6}$ and $\bar{c}_{8}$ values, while $N_{i}^{\mathrm{BKG}}$ is the number of background events in the same bin, for a total of $n_{\text {bins. }}$. The $\theta$ function ensures that if the prediction for $N_{i}^{H H}\left(\bar{c}_{6}, \bar{c}_{8}\right)$ is negative, the information from the bin $i$ is discarded. In our study, since always $\bar{c}_{8}^{\text {true }}=0$, $N_{i}^{H H}\left(\bar{c}_{6}^{\text {true }}, \bar{c}_{8}^{\text {true }}\right)$ is never negative.

Bounds on $\bar{c}_{6}$ and $\bar{c}_{8}$ have been obtained following a fit procedure similar the one presented in ref. [21], from which we have taken also the selection cuts (see appendix A) and binning in the $m(H H)$ distribution. For this reason, the value of $N_{i}^{\mathrm{BKG}}$ is directly taken from ref. [21]. On the contrary, $N_{i}^{H H}\left(\bar{c}_{6}, \bar{c}_{8}\right)$ is derived from the value $N_{i}^{H H}(0,0)$, the SM prediction, from the same reference, which takes into account also higher-order QCD corrections. Assuming that these effect factorise with $\bar{c}_{6}$ and $\bar{c}_{8}$ corrections, the selection cuts of appendix A and the shower effects involved in the simulation of $N_{i}^{H H}(0,0)$, we can obtain $N_{i}^{H H}\left(\bar{c}_{6}, \bar{c}_{8}\right)$ via the relation

$$
N_{i}^{H H}\left(\bar{c}_{6}, \bar{c}_{8}\right)=N_{i}^{H H}(0,0) \mu_{i}^{\text {theory }}\left(\bar{c}_{6}, \bar{c}_{8}\right), \quad \mu_{i}^{\text {theory }} \equiv \frac{\int d \Phi_{i}\left(d \sigma_{\mathrm{NLO}}^{\mathrm{pheno}} / d \Phi_{i}\right)}{\int d \Phi_{i}\left(d \sigma_{\mathrm{LO}}^{\mathrm{SM}} / d \Phi_{i}\right)},
$$

where in the right equation we have understood the dependence on $\bar{c}_{6}$ and $\bar{c}_{8}$ and $\sigma_{\mathrm{LO}}^{\mathrm{SM}}=\left.\sigma_{\mathrm{NLO}}^{\text {pheno }}\right|_{\bar{c}_{6}=0, \bar{c}_{8}=0}$. The quantity $\Phi_{i}$ corresponds to the $b \bar{b} \gamma \gamma$ phase space such that the reconstructed $m(H H)$ value is within the bin $i$. Within all the work, unless differently specified, we take into account the selection cuts of appendix $\mathrm{A}$ in $\Phi_{i}$. When we say "flat 

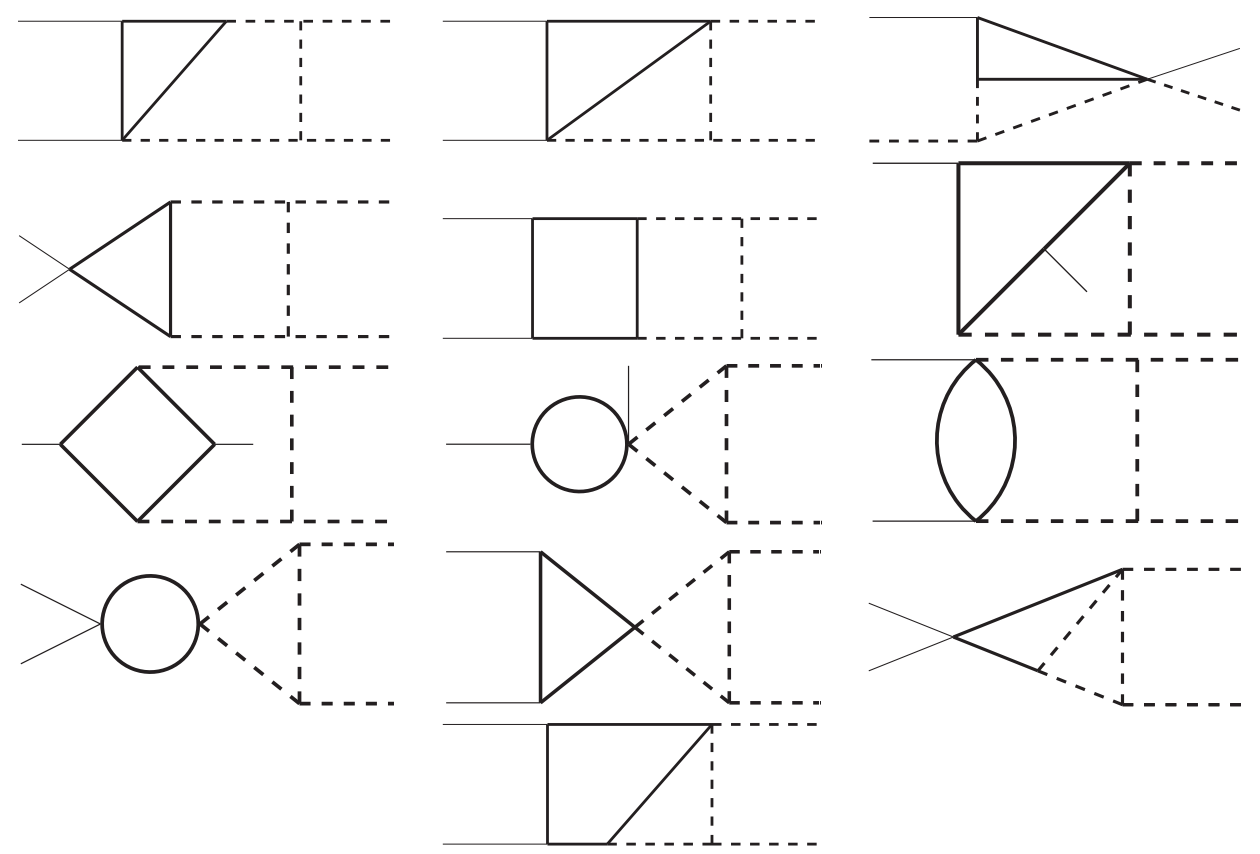

Figure 18. Topologies of the integral expressions from the form factors $\tilde{F}_{0, a}$ and $\tilde{F}_{2, a}$.
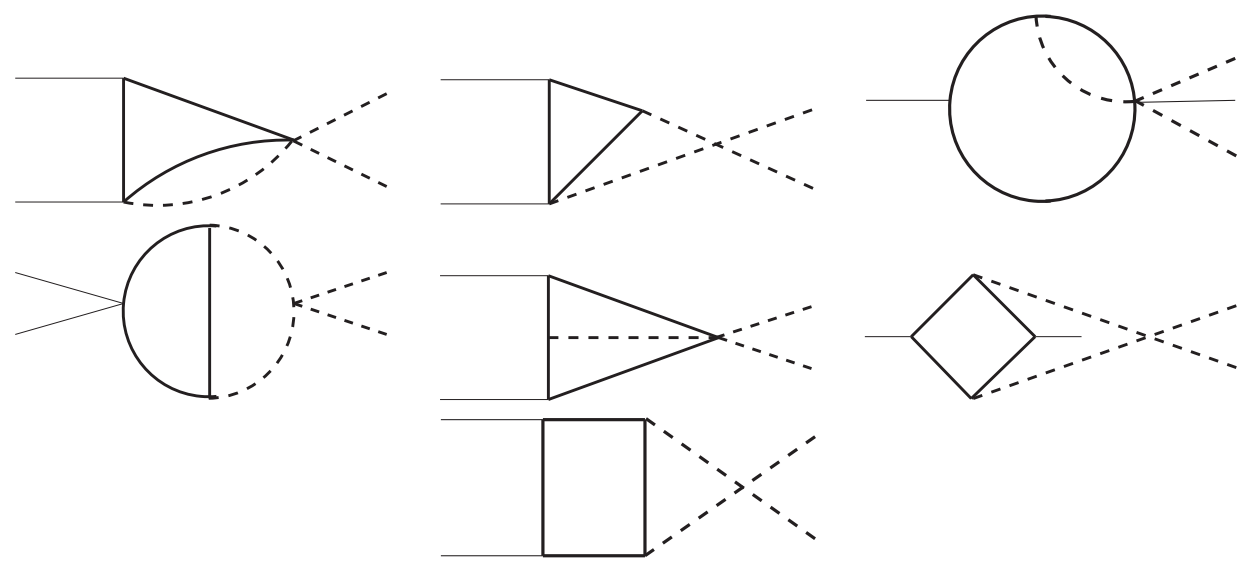

Figure 19. Topologies of the integral expressions from the form factor $\tilde{F}_{0, b}$.

$\mu$-bin" we precisely refer to the case where selection cuts are not taken into account for the definition of $\mu_{i}^{\text {theory }}$.

\section{Topologies of the integral expressions from non-factorisable two-loop contributions}

In this appendix we show the topologies of the integral expressions obtained from nonfactorisable two-loop contributions. Each topology can lead to more than one integral expression. In figure 18 we show those relevant for $\tilde{F}_{0, a}$ and $\tilde{F}_{2, a}$, while in figure 19 those for $\tilde{F}_{0, b}$. Thick lines correspond to massive propagators, dashed with mass $m_{H}$ while solid with mass $m_{t}$. The solid-thin lines corresponds to massless propagators. 
Open Access. This article is distributed under the terms of the Creative Commons Attribution License (CC-BY 4.0), which permits any use, distribution and reproduction in any medium, provided the original author(s) and source are credited.

\section{References}

[1] ATLAS collaboration, Observation of a new particle in the search for the Standard Model Higgs boson with the ATLAS detector at the LHC, Phys. Lett. B 716 (2012) 1 [arXiv: 1207.7214] [INSPIRE].

[2] CMS collaboration, Observation of a new boson at a mass of $125 \mathrm{GeV}$ with the CMS experiment at the LHC, Phys. Lett. B 716 (2012) 30 [arXiv:1207.7235] [INSPIRE].

[3] ATLAS collaboration, Evidence for the spin-0 nature of the Higgs boson using ATLAS data, Phys. Lett. B 726 (2013) 120 [arXiv:1307.1432] [INSPIRE].

[4] CMS collaboration, Measurement of the properties of a Higgs boson in the four-lepton final state, Phys. Rev. D 89 (2014) 092007 [arXiv:1312.5353] [InSPIRE].

[5] ATLAS and CMS collaborations, Measurements of the Higgs boson production and decay rates and constraints on its couplings from a combined ATLAS and CMS analysis of the LHC pp collision data at $\sqrt{s}=7$ and 8 TeV, JHEP 08 (2016) 045 [arXiv: 1606. 02266] [INSPIRE].

[6] CMS collaboration, Combined measurements of Higgs boson couplings in proton-proton collisions at $\sqrt{s}=13 \mathrm{TeV}$, submitted to Eur. Phys. J. (2018) [arXiv:1809.10733] [InSPIRE].

[7] CMS collaboration, Observation of the Higgs boson decay to a pair of $\tau$ leptons with the CMS detector, Phys. Lett. B 779 (2018) 283 [arXiv:1708.00373] [INSPIRE].

[8] ATLAS collaboration, Cross-section measurements of the Higgs boson decaying to a pair of tau leptons in proton-proton collisions at $\sqrt{s}=13 \mathrm{TeV}$ with the ATLAS detector, ATLAS-CONF-2018-021.

[9] CMS collaboration, Observation of Higgs boson decay to bottom quarks, Phys. Rev. Lett. 121 (2018) 121801 [arXiv:1808.08242] [INSPIRE].

[10] ATLAS collaboration, Observation of $H \rightarrow b \bar{b}$ decays and $V H$ production with the ATLAS detector, Phys. Lett. B 786 (2018) 59 [arXiv: 1808.08238] [INSPIRE].

[11] ATLAS collaboration, Observation of Higgs boson production in association with a top quark pair at the LHC with the ATLAS detector, Phys. Lett. B 784 (2018) 173 [arXiv: 1806.00425] [INSPIRE].

[12] CMS collaboration, Observation of $t \bar{t} H$ production, Phys. Rev. Lett. 120 (2018) 231801 [arXiv: 1804.02610] [INSPIRE].

[13] ATLAS collaboration, Search for pair production of Higgs bosons in the b $\bar{b} b \bar{b}$ final state using proton-proton collisions at $\sqrt{s}=13 \mathrm{TeV}$ with the ATLAS detector, JHEP 01 (2019) 030 [arXiv: 1804.06174] [INSPIRE].

[14] ATLAS collaboration, Search for Higgs boson pair production in the $\gamma \gamma b \bar{b}$ final state with 13 TeV pp collision data collected by the ATLAS experiment, JHEP 11 (2018) 040 [arXiv: 1807.04873] [INSPIRE].

[15] ATLAS collaboration, Combination of searches for Higgs boson pairs in pp collisions at $13 \mathrm{TeV}$ with the ATLAS experiment, ATLAS-CONF-2018-043. 
[16] ATLAS collaboration, Search for resonant and non-resonant Higgs boson pair production in the $b \bar{b} \tau^{+} \tau^{-}$decay channel in pp collisions at $\sqrt{s}=13 \mathrm{TeV}$ with the ATLAS detector, Phys. Rev. Lett. 121 (2018) 191801 [arXiv:1808.00336] [INSPIRE].

[17] U. Baur, T. Plehn and D.L. Rainwater, Probing the Higgs selfcoupling at hadron colliders using rare decays, Phys. Rev. D 69 (2004) 053004 [hep-ph/0310056] [INSPIRE].

[18] J. Baglio, A. Djouadi, R. Gröber, M.M. Mühlleitner, J. Quevillon and M. Spira, The measurement of the Higgs self-coupling at the LHC: theoretical status, JHEP 04 (2013) 151 [arXiv: 1212.5581] [INSPIRE].

[19] W. Yao, Studies of measuring Higgs self-coupling with $H H \rightarrow b \bar{b} \gamma \gamma$ at the future hadron colliders, in Proceedings, 2013 Community Summer Study on the Future of U.S. Particle Physics: Snowmass on the Mississippi (CSS2013), Minneapolis, MN, U.S.A., July 29-August 6, 2013 (2013) [arXiv: 1308.6302] [INSPIRE].

[20] V. Barger, L.L. Everett, C.B. Jackson and G. Shaughnessy, Higgs-Pair Production and Measurement of the Triscalar Coupling at LHC(8,14), Phys. Lett. B 728 (2014) 433 [arXiv: 1311.2931] [INSPIRE].

[21] A. Azatov, R. Contino, G. Panico and M. Son, Effective field theory analysis of double Higgs boson production via gluon fusion, Phys. Rev. D 92 (2015) 035001 [arXiv:1502.00539] [INSPIRE].

[22] C.-T. Lu, J. Chang, K. Cheung and J.S. Lee, An exploratory study of Higgs-boson pair production, JHEP 08 (2015) 133 [arXiv:1505. 00957] [INSPIRE].

[23] M.J. Dolan, C. Englert and M. Spannowsky, Higgs self-coupling measurements at the LHC, JHEP 10 (2012) 112 [arXiv:1206.5001] [INSPIRE].

[24] A. Papaefstathiou, L.L. Yang and J. Zurita, Higgs boson pair production at the LHC in the $b \bar{b} W^{+} W^{-}$channel, Phys. Rev. D 87 (2013) 011301 [arXiv:1209.1489] [inSPIRE].

[25] D.E. Ferreira de Lima, A. Papaefstathiou and M. Spannowsky, Standard model Higgs boson pair production in the $(b \bar{b})(b \bar{b})$ final state, JHEP 08 (2014) 030 [arXiv:1404.7139] [INSPIRE].

[26] D. Wardrope, E. Jansen, N. Konstantinidis, B. Cooper, R. Falla and N. Norjoharuddeen, Non-resonant Higgs-pair production in the $b \bar{b} b \bar{b}$ final state at the LHC, Eur. Phys. J. C 75 (2015) 219 [arXiv: 1410.2794] [INSPIRE].

[27] J.K. Behr, D. Bortoletto, J.A. Frost, N.P. Hartland, C. Issever and J. Rojo, Boosting Higgs pair production in the $b \bar{b} b \bar{b}$ final state with multivariate techniques, Eur. Phys. J. C 76 (2016) 386 [arXiv: 1512.08928] [INSPIRE].

[28] C. Englert, F. Krauss, M. Spannowsky and J. Thompson, Di-Higgs phenomenology in t $\bar{t} h h$ : The forgotten channel, Phys. Lett. B 743 (2015) 93 [arXiv:1409.8074] [InSPIRE].

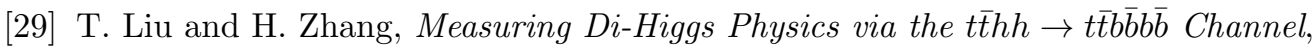
arXiv:1410.1855 [INSPIRE].

[30] Q.-H. Cao, Y. Liu and B. Yan, Measuring trilinear Higgs coupling in WHH and ZHH productions at the high-luminosity LHC, Phys. Rev. D 95 (2017) 073006 [arXiv: 1511.03311] [INSPIRE].

[31] C. Englert, R. Kogler, H. Schulz and M. Spannowsky, Higgs coupling measurements at the LHC, Eur. Phys. J. C 76 (2016) 393 [arXiv:1511.05170] [INSPIRE]. 
[32] F. Bishara, R. Contino and J. Rojo, Higgs pair production in vector-boson fusion at the LHC and beyond, Eur. Phys. J. C 77 (2017) 481 [arXiv:1611.03860] [INSPIRE].

[33] Q.-H. Cao, G. Li, B. Yan, D.-M. Zhang and H. Zhang, Double Higgs production at the $14 \mathrm{TeV}$ LHC and a $100 \mathrm{TeV}$ pp collider, Phys. Rev. D 96 (2017) 095031 [arXiv:1611.09336] [INSPIRE].

[34] T. Huang et al., Resonant di-Higgs boson production in the $b \bar{b} W W$ channel: Probing the electroweak phase transition at the LHC, Phys. Rev. D 96 (2017) 035007 [arXiv: 1701.04442] [inSPIRE].

[35] A. Adhikary, S. Banerjee, R.K. Barman, B. Bhattacherjee and S. Niyogi, Revisiting the non-resonant Higgs pair production at the HL-LHC, JHEP 07 (2018) 116 [arXiv: 1712.05346] [INSPIRE].

[36] D. Gonçalves, T. Han, F. Kling, T. Plehn and M. Takeuchi, Higgs boson pair production at future hadron colliders: From kinematics to dynamics, Phys. Rev. D 97 (2018) 113004 [arXiv: 1802.04319] [INSPIRE].

[37] J. Chang, K. Cheung, J.S. Lee, C.-T. Lu and J. Park, Higgs-boson-pair production $H(\rightarrow b \bar{b}) H(\rightarrow \gamma \gamma)$ from gluon fusion at the HL-LHC and HL-100 TeV hadron collider, arXiv: 1804.07130 [INSPIRE].

[38] E. Arganda, C. Garcia-Garcia and M.J. Herrero, Probing the Higgs self-coupling through double Higgs production in vector boson scattering at the LHC, arXiv:1807.09736 [INSPIRE].

[39] S. Homiller and P. Meade, Measurement of the Triple Higgs Coupling at a HE-LHC, arXiv: 1811.02572 [INSPIRE].

[40] S. Borowka et al., Higgs Boson Pair Production in Gluon Fusion at Next-to-Leading Order with Full Top-Quark Mass Dependence, Phys. Rev. Lett. 117 (2016) 012001 [Erratum ibid. 117 (2016) 079901] [arXiv: 1604.06447] [INSPIRE].

[41] S. Borowka et al., Full top quark mass dependence in Higgs boson pair production at NLO, JHEP 10 (2016) 107 [arXiv: 1608.04798] [INSPIRE].

[42] J. Baglio, F. Campanario, S. Glaus, M. Mühlleitner, M. Spira and J. Streicher, Gluon fusion into Higgs pairs at NLO QCD and the top mass scheme, arXiv:1811.05692 [INSPIRE].

[43] R. Frederix et al., Higgs pair production at the LHC with NLO and parton-shower effects, Phys. Lett. B 732 (2014) 142 [arXiv:1401.7340] [INSPIRE].

[44] G. Heinrich, S.P. Jones, M. Kerner, G. Luisoni and E. Vryonidou, NLO predictions for Higgs boson pair production with full top quark mass dependence matched to parton showers, JHEP 08 (2017) 088 [arXiv: 1703.09252] [INSPIRE].

[45] S. Jones and S. Kuttimalai, Parton Shower and NLO-Matching uncertainties in Higgs Boson Pair Production, JHEP 02 (2018) 176 [arXiv:1711.03319] [INSPIRE].

[46] D. de Florian and J. Mazzitelli, Higgs Boson Pair Production at Next-to-Next-to-Leading Order in QCD, Phys. Rev. Lett. 111 (2013) 201801 [arXiv:1309.6594] [INSPIRE].

[47] J. Grigo, K. Melnikov and M. Steinhauser, Virtual corrections to Higgs boson pair production in the large top quark mass limit, Nucl. Phys. B 888 (2014) 17 [arXiv:1408.2422] [INSPIRE].

[48] J. Grigo, J. Hoff and M. Steinhauser, Higgs boson pair production: top quark mass effects at NLO and NNLO, Nucl. Phys. B 900 (2015) 412 [arXiv:1508.00909] [INSPIRE]. 
[49] D. de Florian et al., Differential Higgs Boson Pair Production at Next-to-Next-to-Leading Order in QCD, JHEP 09 (2016) 151 [arXiv: 1606.09519] [INSPIRE].

[50] M. Grazzini et al., Higgs boson pair production at NNLO with top quark mass effects, JHEP 05 (2018) 059 [arXiv: 1803.02463] [INSPIRE].

[51] T. Plehn and M. Rauch, The quartic Higgs coupling at hadron colliders, Phys. Rev. D 72 (2005) 053008 [hep-ph/0507321] [INSPIRE].

[52] T. Binoth, S. Karg, N. Kauer and R. Ruckl, Multi-Higgs boson production in the Standard Model and beyond, Phys. Rev. D 74 (2006) 113008 [hep-ph/0608057] [InSPIRE].

[53] C.-Y. Chen, Q.-S. Yan, X. Zhao, Y.-M. Zhong and Z. Zhao, Probing triple-Higgs productions via $4 b 2 \gamma$ decay channel at a $100 \mathrm{TeV}$ hadron collider, Phys. Rev. D 93 (2016) 013007 [arXiv: 1510.04013] [INSPIRE].

[54] W. Kilian, S. Sun, Q.-S. Yan, X. Zhao and Z. Zhao, New Physics in multi-Higgs boson final states, JHEP 06 (2017) 145 [arXiv:1702.03554] [INSPIRE].

[55] B. Fuks, J.H. Kim and S.J. Lee, Scrutinizing the Higgs quartic coupling at a future $100 \mathrm{TeV}$ proton-proton collider with taus and b-jets, Phys. Lett. B 771 (2017) 354 [arXiv: 1704.04298] [INSPIRE].

[56] R. Contino et al., Physics at a $100 \mathrm{TeV}$ pp collider: Higgs and EW symmetry breaking studies, CERN Yellow Report (2017) 255 [arXiv: 1606.09408] [INSPIRE].

[57] F. Maltoni, E. Vryonidou and M. Zaro, Top-quark mass effects in double and triple Higgs production in gluon-gluon fusion at NLO, JHEP 11 (2014) 079 [arXiv:1408.6542] [INSPIRE].

[58] M. Spira, Effective Multi-Higgs Couplings to Gluons, JHEP 10 (2016) 026 [arXiv: 1607.05548] [INSPIRE].

[59] D. de Florian and J. Mazzitelli, Two-loop corrections to the triple Higgs boson production cross section, JHEP 02 (2017) 107 [arXiv:1610.05012] [INSPIRE].

[60] M. McCullough, An Indirect Model-Dependent Probe of the Higgs Self-Coupling, Phys. Rev. D 90 (2014) 015001 [Erratum ibid. D 92 (2015) 039903] [arXiv:1312.3322] [INSPIRE].

[61] M. Gorbahn and U. Haisch, Indirect probes of the trilinear Higgs coupling: $g g \rightarrow h$ and $h \rightarrow \gamma \gamma$, JHEP 10 (2016) 094 [arXiv: 1607.03773] [INSPIRE].

[62] G. Degrassi, P.P. Giardino, F. Maltoni and D. Pagani, Probing the Higgs self coupling via single Higgs production at the LHC, JHEP 12 (2016) 080 [arXiv:1607.04251] [INSPIRE].

[63] W. Bizon, M. Gorbahn, U. Haisch and G. Zanderighi, Constraints on the trilinear Higgs coupling from vector boson fusion and associated Higgs production at the LHC, JHEP 07 (2017) 083 [arXiv: 1610.05771] [INSPIRE].

[64] F. Maltoni, D. Pagani, A. Shivaji and X. Zhao, Trilinear Higgs coupling determination via single-Higgs differential measurements at the LHC, Eur. Phys. J. C 77 (2017) 887 [arXiv: 1709.08649] [INSPIRE].

[65] S. Di Vita, C. Grojean, G. Panico, M. Riembau and T. Vantalon, A global view on the Higgs self-coupling, JHEP 09 (2017) 069 [arXiv:1704.01953] [INSPIRE].

[66] T. Barklow, K. Fujii, S. Jung, M.E. Peskin and J. Tian, Model-Independent Determination of the Triple Higgs Coupling at $e^{+} e^{-}$Colliders, Phys. Rev. D 97 (2018) 053004 [arXiv: 1708.09079] [INSPIRE]. 
[67] S. Di Vita et al., A global view on the Higgs self-coupling at lepton colliders, JHEP 02 (2018) 178 [arXiv: 1711.03978] [INSPIRE].

[68] F. Maltoni, D. Pagani and X. Zhao, Constraining the Higgs self-couplings at $e^{+} e^{-}$colliders, JHEP 07 (2018) 087 [arXiv: 1802.07616] [INSPIRE].

[69] G. Degrassi, M. Fedele and P.P. Giardino, Constraints on the trilinear Higgs self coupling from precision observables, JHEP 04 (2017) 155 [arXiv: 1702.01737] [INSPIRE].

[70] G.D. Kribs, A. Maier, H. Rzehak, M. Spannowsky and P. Waite, Electroweak oblique parameters as a probe of the trilinear Higgs boson self-interaction, Phys. Rev. D 95 (2017) 093004 [arXiv: 1702.07678] [INSPIRE].

[71] T. Liu, K.-F. Lyu, J. Ren and H.X. Zhu, Probing the quartic Higgs boson self-interaction, Phys. Rev. D 98 (2018) 093004 [arXiv: 1803.04359] [InSPIRE].

[72] W. Bizon, U. Haisch and L. Rottoli, Constraints on the quartic Higgs self-coupling from double-Higgs production at future hadron colliders, arXiv:1810.04665 [INSPIRE].

[73] S. Borowka et al., pySecDec: a toolbox for the numerical evaluation of multi-scale integrals, Comput. Phys. Commun. 222 (2018) 313 [arXiv:1703.09692] [INSPIRE].

[74] S. Borowka, G. Heinrich, S. Jahn, S.P. Jones, M. Kerner and J. Schlenk, Numerical evaluation of two-loop integrals with pySecDec, Acta Phys. Polon. Supp. 11 (2018) 375 [arXiv: 1712.05755] [INSPIRE].

[75] A.J. Barr, M.J. Dolan, C. Englert, D.E. Ferreira de Lima and M. Spannowsky, Higgs Self-Coupling Measurements at a 100 TeV Hadron Collider, JHEP 02 (2015) 016 [arXiv: 1412.7154] [INSPIRE].

[76] H.--J. He, J. Ren and W. Yao, Probing new physics of cubic Higgs boson interaction via Higgs pair production at hadron colliders, Phys. Rev. D 93 (2016) 015003 [arXiv:1506.03302] [INSPIRE].

[77] M.L. Mangano et al., Physics at a $100 \mathrm{TeV}$ pp Collider: Standard Model Processes, CERN Yellow Report (2017) 1 [arXiv: 1607.01831] [INSPIRE].

[78] F. Boudjema and E. Chopin, Double Higgs production at the linear colliders and the probing of the Higgs selfcoupling, Z. Phys. C 73 (1996) 85 [hep-ph/9507396] [INSPIRE].

[79] S. Dittmaier, A. Huss and C. Schwinn, Dominant mixed QCD-electroweak $O\left(\alpha_{s} \alpha\right)$ corrections to Drell-Yan processes in the resonance region, Nucl. Phys. B 904 (2016) 216 [arXiv: 1511.08016] [INSPIRE].

[80] M. Bonetti, K. Melnikov and L. Tancredi, Higher order corrections to mixed QCD-EW contributions to Higgs boson production in gluon fusion, Phys. Rev. D 97 (2018) 056017 [Erratum ibid. D 97 (2018) 099906] [arXiv:1801.10403] [INSPIRE].

[81] C. Anastasiou et al., Mixed QCD-electroweak corrections to Higgs production via gluon fusion in the small mass approximation, arXiv:1811.11211 [INSPIRE].

[82] L. Di Luzio, R. Gröber and M. Spannowsky, Maxi-sizing the trilinear Higgs self-coupling: how large could it be?, Eur. Phys. J. C 77 (2017) 788 [arXiv:1704.02311] [INSPIRE].

[83] P. Nogueira, Automatic Feynman graph generation, J. Comput. Phys. 105 (1993) 279.

[84] J.A.M. Vermaseren, New features of FORM, math-ph/0010025 [INSPIRE]. 
[85] G. Degrassi, P.P. Giardino and R. Gröber, On the two-loop virtual QCD corrections to Higgs boson pair production in the Standard Model, Eur. Phys. J. C 76 (2016) 411 [arXiv: 1603.00385] [INSPIRE].

[86] S. Borowka, G. Heinrich, S.P. Jones, M. Kerner, J. Schlenk and T. Zirke, SecDec-3.0: numerical evaluation of multi-scale integrals beyond one loop, Comput. Phys. Commun. 196 (2015) 470 [arXiv: 1502.06595] [INSPIRE].

[87] J. Berntsen, T.O. Espelid and A. Genz, Algorithm 698: DCUHRE: An adaptive multidimensional integration routine for a vector of integrals. ACM Trans. Math. Software 17 (1991) 452.

[88] T. Hahn, CUBA: A Library for multidimensional numerical integration, Comput. Phys. Commun. 168 (2005) 78 [hep-ph/0404043] [INSPIRE].

[89] G.P. Lepage, A New Algorithm for Adaptive Multidimensional Integration, J. Comput. Phys. 27 (1978) 192 [INSPIRE].

[90] M. Jacob and G.C. Wick, On the general theory of collisions for particles with spin, Annals Phys. 7 (1959) 404 [INSPIRE].

[91] S. Dulat et al., New parton distribution functions from a global analysis of quantum chromodynamics, Phys. Rev. D 93 (2016) 033006 [arXiv: 1506.07443] [INSPIRE].

[92] ATLAS collaboration, Study of the double Higgs production channel $H(\rightarrow b \bar{b}) H(\rightarrow \gamma \gamma)$ with the ATLAS experiment at the HL-LHC, ATL-PHYS-PUB-2017-001.

[93] M.L. Mangano, T. Plehn, P. Reimitz, T. Schell and H.-S. Shao, Measuring the Top Yukawa Coupling at $100 \mathrm{TeV}, \mathrm{J}$. Phys. G 43 (2016) 035001 [arXiv:1507.08169] [inSPIRE].

[94] A. Papaefstathiou and K. Sakurai, Triple Higgs boson production at a $100 \mathrm{TeV}$ proton-proton collider, JHEP 02 (2016) 006 [arXiv:1508.06524] [INSPIRE].

[95] CMS collaboration, Constraints on the Higgs boson self-coupling from ttH $+t H, H \rightarrow \gamma \gamma$ differential measurements at the HL-LHC, CMS-PAS-FTR-18-020. 